\title{
La hipótesis en la lengua de señas colombiana (LSC) ${ }^{1}$
}

\author{
Lionel Antonio Tovar (1) \\ Lorenzo López (1) \\ Universidad del Valle \\ Cali, Colombia
}

\section{Resumen}

En este artículo se continúa con la exploración de las funciones del lenguaje académico en la lengua de señas colombiana (LSC), iniciada por los mismos autores. El propósito del estudio es presentar la estructura de la hipótesis en LSC, tal como aparece en textos producidos por informantes sordos durante discusiones metalingüísticas suscitadas a partir de textos escritos de hipótesis en español. Primero se presenta el sistema de la modalización de probabilidad y su estructura léxico-gramatical en español, así como el papel de la gestualidad en su expresión. Seguidamente se presenta la modalización de probabilidad en LSC y se dan ejemplos de textos de hipótesis producidos por los señantes, para ilustrar y analizar la léxico-gramática de esta función.

Palabras clave: lengua de señas colombiana (LSC); política y planificación lingüística; lenguas de señas; discurso académico; signalidad académica; hipótesis; educación de Sordos; educación bilingüe de Sordos.

\section{Abstract}

\section{Hypothesis in Colombian Sign Language (LSC)}

This article continues the exploration of academic language functions in the Colombian Sign Language (LSC), initiated by the authors in previous articles. The purpose of the study is to describe the structure of hypothesis in LSC as it appears in texts produced by Deaf informants during metalinguistic discussions raised from written texts of hypothesis in Spanish. First, the system of probability modalization is presented along with its lexico-grammatical structure in Spanish and the role of gesture in its expression. Then probability modalization in LSC is presented and

\footnotetext{
${ }^{1}$ Este artículo presenta los hallazgos sobre hipótesis en la investigación "Modalidades discursivas académicas en la lengua de señas colombiana (LSC)", registrada por el autor principal como investigación del Grupo de Investigación en Bilingüismo, código CI-4333, Vicerrectoría de Investigaciones, Universidad del Valle, Cali.

El autor principal agradece a sus informantes sordos, Rubén Darío Pichicá, Angélica Muñoz y Cristhian Sepúlveda, así como a su coautor, Lorenzo López Gómez, quien lo apoyó sustancialmente en la transcripción y en la identificación de la marcación de la hipótesis.
} 
examples are given of hypothesis texts produced by the signers, to illustrate and analyze the lexico-grammar of this function.

Key words: Colombian Sign Language (LSC); language planning and policy; sign language; academic discourse; academic signacy; hypothesis; Deaf education; bilingual education for the Deaf.

\section{Résumé}

\section{L'hypothèse en langue des signes colombienne (LSC)}

Cet article poursuit l'exploration des fonctions du langage académique dans la langue de signes colombienne (LSC), initiée par les auteurs eux-mêmes. Le but de l'étude est de présenter la structure de l'hypothèse en LSC, tel qu'elle apparaît dans des textes produits par les informateurs sourds lors de discussions métalinguistiques issues de textes écrits d'hypothèse en espagnol. Tout d'abord le système de la modalisation de probabilité est présenté ainsi que sa structure léxico-grammaticale en espagnol et le rôle du geste dans son expression. Ensuite, des exemples de textes d'hypothèse produits par les signants sont donnés, afin d'illustrer et analyser la léxico-grammaire de cette fonction.

Mots-clés: langue des signes colombienne (LSC); politique et aménagement linguistique; langues des signes; discours académique; signacie académique; hypothèse ; éducation des sourds ; éducation bilingue des sourds. 


\section{SOBRE LOS AUTORES}

\section{Lionel Antonio Tovar}

Doctor en Lingüística, Universidad de Los Andes, Mérida, Venezuela; M.A. en Lingüística, University of Kansas, EE.UU. Sus áreas de docencia e investigación actuales incluyen la enseñanza y adquisición de segundas lenguas, el bilingüismo y la educación bilingüe, y la planificación lingüística de lenguas minoritarias. Su trabajo se ha orientado en estas áreas principalmente hacia las necesidades de la comunidad de sordos colombiana y su lengua de señas colombiana (LSC). Actualmente es Vicedecano de Investigación y Posgrado de la Facultad de Humanidades de la Universidad del Valle, y Director del Doctorado en Humanidades en la misma universidad.

Correo electrónico: lionel.tovar@correounivalle.edu.co

\section{Lorenzo López}

Magíster en Estudios Interlingüísticos e Interculturales, Universidad del Valle; Licenciado en Español y Comunicación, Universidad de Pamplona. Tiene amplia experiencia como traductor/intérprete de lengua de señas colombiana (LSC) y en los últimos cinco años se ha desempeñado como profesor de Modalidades Discursivas en LSC y Práctica de Interpretación en el programa de Tecnología en Interpretación para Sordos y Sordociegos de la Universidad del Valle. Ha hecho investigación en traducción/interpretación bimodal. Actualmente es el representante legal de la Federación Nacional de Traductores, Intérpretes y Guías Intérpretes de Lengua de Señas de Colombia (FENILC).

Correo electrónico: lorenzo.lopez@correounivalle.edu.co

\section{CÓMO CITAR ESTE ARTÍCULO}

Tovar, L., \& López, L. (2021). La hipótesis en la lengua de señas colombiana (LSC). Lenguaje, 49(2), 231-274. https://doi.org/10.25100/lenguaje.v49i2.6524. 


\section{INTRODUCCIÓN}

La hipótesis es una de las funciones comunicativas académicas más importantes. En las ciencias, los estudiosos tienen preguntas de investigación o problemas de investigación para los cuales quieren encontrar una respuesta. Muy a menudo, lo primero que hacen, a partir de sus observaciones, es dar una explicación provisional para tratar de entender lo que pasa. Una hipótesis es, entonces, una explicación provisional de un hecho o situación, que puede ser después probada o descartada con base en evidencias o pruebas que se hagan. Cuando una hipótesis queda probada, deja de ser hipótesis y se convierte en un hecho comprobado. No siempre, sin embargo, ese hecho comprobado es aceptado definitivamente, pues con nuevas teorías hay nuevas explicaciones.

Pero esto mismo pasa en la conversación cotidiana tanto en lenguas orales, en la modalidad auditivo-vocal, como en lenguas de señas, que utilizan en cambio la modalidad viso-gestual y expresan los mensajes mediante articuladores claramente visibles de la parte anterior superior del cuerpo (cabeza, tronco, brazos, manos, dedos y cara). También en esta comunicación interpersonal básica a menudo estamos frente a situaciones y hacemos hipótesis o conjeturas acerca de lo que puede estar pasando. En ambos tipos de lenguas, en la comunicación cara a cara, juega un papel importante el contexto situacional y las personas se dan cuenta de que lo que expresamos es una hipótesis no solo por lo que decimos, sino también por cómo lo decimos: En las 234 lenguas orales, acompañando el enunciado con la prosodia y los gestos; en las lenguas de señas, acompañando el enunciado con gestos (que se dan en la misma modalidad viso-gestual), algunos de los cuales tienen su equivalente en la prosodia y la gestualidad que acompaña la expresión en lenguas orales. Como se verá, en el caso de la hipótesis en la lengua de señas, hay que distinguir, aunque no siempre será fácil, entre esta gestualidad y los rasgos no manuales que asumen una función léxicogramatical.

De hecho, como resalta Matthiessen (2004), el sistema de la POLARIDAD es muy probablemente uno de los sistemas más altamente gramaticalizados en todas las lenguas, aun cuando sus formas de realización pueden variar considerablemente.

\section{Antecedentes}

Varios autores de lingüística en la modalidad viso-gestual han tratado aspectos gramaticales que sirven para marcar la hipótesis en diversas lenguas de señas, sobre todo prestando atención a las marcaciones que aparecen en el Grupo Verbal (por ejemplo, Baker et al., 2016; Hill et al., 2019; Johnston y Schembri, 2007; Liddell, 1980, 2003; Neidle et al., 2000; Padden, 1988; Pfau et al., 2012; Sandler, 2012; Sandler y LilloMartin, 2006; Sutton-Spence y Woll, 1998; Valli et al., 2011; Wilbur, 1987; Wilcox et al., 2010). Sin embargo, los tratamientos son por lo regular fragmentarios $y$, sobre todo, en ninguno de ellos se concentra la atención en la hipótesis como función comunicativa, conjugando aspectos léxicos y gramaticales que atañen a toda la 
cláusula y no solo al Grupo Verbal. Es por ello que se ha elegido un modelo más amplio, la Gramática Sistémico-Funcional, en el que se refleja más claramente la relación entre las selecciones léxico-gramaticales y el discurso.

\section{MARCO TEÓRICO}

\section{La hipótesis según la Gramática Sistémico-Funcional}

Se presenta aquí sucintamente la descripción de la hipótesis desde el punto de vista de la Gramática Sistémico-Funcional de Halliday (2014), con ejemplos en español, de modo que al lector de esta lengua le quede más fácil entender este modelo. La hipótesis, según Halliday (2014), hace parte de la metafunción interpersonal, es decir, la que trata de los recursos que nos permiten iniciar y mantener la comunicación, establecer el propósito de la interacción y la relación entre sus participantes, y expresar nuestras actitudes o creencias. El sistema principal en esta metafunción es el sistema del MODO. Según Halliday (2014), los enunciados se distribuyen en cuatro funciones del habla: proveer o solicitar información (proposiciones) o proveer o solicitar bienes y servicios (propuestas), como se ve en la Tabla 1:

Tabla 1. Tipos de funciones del habla

\begin{tabular}{|l|l|l|}
\hline \multicolumn{1}{|c|}{$\begin{array}{c}\text { Papel en el } \\
\text { intercambio }\end{array}$} & Producto que se intercambia \\
\cline { 2 - 3 } & (a) bienes y servicios & (b) información \\
\hline (i) proveer & 'ofrecimiento' & 'declarativa' \\
& ¿quieres esta tetera? & él le está dando a ella la tetera. \\
\hline (ii) solicitar & 'orden' & 'pregunta' \\
& idame la tetera! & ¿qué le está dando él a ella? \\
\hline
\end{tabular}

Fuente: Traducida de Halliday (2014, p. 136)

En el análisis interpersonal, la estructura de las cláusulas, que son generalmente declarativas, cuya función es precisamente proveer información, presenta los siguientes elementos: Sujeto, Finito u Operador Finito, Predicador, Complemento y Adjunto Circunstancial. El Sujeto y el Finito, por su parte, funcionan en muchas lenguas como un solo elemento, denominado el Modo. El resto de los elementos de la cláusula forman lo que se denomina el Residuo. Todo esto se ejemplifica en la Tabla 2: 
Tabla 2. Estructura de la cláusula según la metafunción interpersonal

\begin{tabular}{|c|c|c|c|c|}
\hline \multirow[t]{2}{*}{ Los maestros } & \multicolumn{2}{|c|}{ levantaron ${ }^{2}$} & \multirow[t]{2}{*}{ el paro } & \multirow[t]{2}{*}{ tras acuerdo con el gobierno } \\
\hline & -aron & levant- & & \\
\hline Sujeto & Finito & Predicador & Complemento & $\begin{array}{c}\text { Adjunto Circunstancial: } \\
\text { Causa/Tiempo }\end{array}$ \\
\hline \multicolumn{2}{|c|}{ Modo } & \multicolumn{3}{|c|}{ Residuo } \\
\hline
\end{tabular}

Según el mismo Halliday (2014), el Sujeto es algo con referencia a lo cual la proposición puede afirmarse o negarse, por lo que es siempre un Grupo Nominal. El Finito y el Predicador hacen parte de un mismo Grupo Verbal. El Finito (llamado a veces "Operador Finito") es el que marca los sistemas de TIEMPO verbal primario (pasado, presente o futuro) POLARIDAD (positiva o negativa), CONTRASTE (neutro o enfático) y MODALIDAD (que se amplía más adelante). Como se ha visto, ambos elementos constituyen la estructura del Modo. El Modo se identifica más fácilmente en lenguas como el inglés, pues se lo retoma en las preguntas de verificación denominadas tag questions: Por ejemplo: Juan is a Colombian, isn't he? Es también el elemento que a menudo sufre elipsis en esa lengua, pues se sobreentiende toda la información provista normalmente por el Finito: Going home? (por Are you going home?). Asimismo es en inglés la base del imperativo: Go! En español, el Sujeto y el Finito concuerdan en persona y número, por lo que a menudo el Finito puede ir sin Sujeto o, incluso, solo con el Finito modal: ¿Vienes? ¿Quieres (hacerlo)? En lugar de las tag questions, necesitamos verificativos como ¿cierto?, ¿verdad? o un marcador de la polaridad como ¿sí?, ¿no? El Predicador es un Grupo Verbal que puede aparecer sin el Finito o unido a él, y su función es especificar el Proceso o verbo, el Tiempo primario con respecto al Tiempo secundario, la Voz (activa o pasiva) y otros aspectos o fases (como comenzar a, esperar, tratar de, ir $a$, etc.). Algunas veces, el Finito se funde con el Predicador en una forma supletiva: es (Predicador ser + Finito 3-per-sg-presindicativo), fue (Predicador ser o ir + Finito 3-per-sg-pret-indicativo). El Complemento es, por su parte, un elemento del Residuo con el potencial de ser Sujeto, pero no lo es, por lo que es normalmente un Grupo Nominal o un Grupo Adjetival. Finalmente, el Adjunto Circunstancial o, simplemente, Adjunto, es el elemento del Residuo que no puede ser Sujeto y está constituido por un Grupo Adverbial o por una Frase Preposicional.

Normalmente, en la conversación diaria y en los textos expositivos, aparece abundante información factual como la del ejemplo de la Tabla 2, es decir que se presenta en forma de afirmaciones que se espera se tomen por ciertas. Pero también aparece información que no es cierta, sino que se presenta aún como hipótesis.

La hipótesis hace parte, más específicamente, del sistema de la MODALIDAD. Con este sistema, los hablantes pueden expresar diferentes grados de proximidad a los polos del continuo entre la Polaridad positiva y la Polaridad negativa que se pueden dar para indicar matices en la significación. Estos continuos los subdivide Halliday

\footnotetext{
2 Para indicar la estructura del Modo, ha habido que subdividir el Grupo Verbal de una manera que es artificial en español, ya que levant- es el Predicador y -aron es el Finito.
} 
(2014) en los de "probabilidad" (probability) y "habitualidad" (usuality), que se dan en las proposiciones, que sirven para las funciones de solicitar o proveer información, y reciben el nombre de subsistema de la MODALIZACIÓN; y en los de "disposición" (willingness) y “obligación” (obligation), que sirven para las funciones de solicitar o proveer bienes y servicios, y que reciben el nombre de subsistema de la MODULACIÓN. La hipótesis pertenece entonces al sistema de la MODALIZACIÓN, que expresa probabilidad de una proposición determinada.

Siguiendo a Lock (1996), se considera aquí la MODALIDAD de manera amplia, lo que puede incluir también aspectos de EVIDENCIALIDAD. Como señala Halliday (2014), “algunas lenguas le dan más prominencia a la EVIDENCIALIDAD que a la MODALIDAD y otras no agrupan MODALIZACIÓN y MODULACIÓN en un sistema unificado" (p. 183). Se puede añadir aquí que la utilización simultánea de la prosodia y la gestualidad complica aún más las cosas. Otra puntualización importante es la señalada por Thompson (2014), quien hace ver que la marcación de la probabilidad, al igual que otras marcaciones propias de la función interpersonal, si bien se puede centrar en la estructura del Modo, se reparte a menudo a lo largo de la cláusula. Los siguientes ejemplos muestran varias posibilidades, que se multiplican si les añadimos cambios prosódicos y gestualidad:

Va a haber un apagón. (cláusula sin MODALIDAD, Polaridad positiva)

Dicen que habrá un apagón. (EVIDENCIALIDAD)

El Ministro de Energía anuncia que habrá un apagón. (EVIDENCIALIDAD)

Podría haber un apagón. (probabilidad indicada por el Finito)

Es posible que haya un apagón. (probabilidad indicada por un Adjunto Modal)

Tal vez haya un apagón. (probabilidad indicada por un Adjunto Modal)

En la universidad dicen que el Ministro de Energía anunció que es posible que haya un apagón, pero no es seguro. (combinación de varias posibilidades)

Según Lavid et al. (2010), hay tres tipos de marcación de la probabilidad en español:

- verbos modales, que a menudo indican también obligación ${ }^{3}$;

- adjetivos, adverbios o sustantivos relacionados con los sentidos de "certeza" o "probabilidad/posibilidad"; según Halliday (2014) y Thompson (2014), estas categorías de procesos se pueden deslindar en categorías más detalladas, como "creencia", "sugerencia", "expectativa", "presunción", "predicción", "hipótesis" o "afirmación temeraria", e incluyen también verbos.

\footnotetext{
${ }^{3}$ Lavid et al. (2010) señalan que rara vez hay ambigüedad en estos casos, ya que la modalidad de obligación se aplica a propuestas y no a proposiciones, por lo que el proceso en una cláusula de obligación tiende a recibir una interpretación perfectiva en situaciones dinámicas, que es normalmente incompatible con una proposición. Así, una situación dinámica en infinitivo le impone a tener que un significado de obligación: Tiene que comer; mientras que un infinitivo en el aspecto continuo se asocia con el significado de probabilidad: Tiene que estar comiendo.
} 
- formas verbales normalmente asociadas con la expresión de la relación temporal de posterioridad, es decir el futuro y el condicional.

Asimismo, la probabilidad se marca con otro tipo de Adjunto que expresa la MODALIDAD, en este caso el subtipo de la MODALIZACIÓN de probabilidad: los Adjuntos Modales. Estos se subdividen en dos grupos: Adjuntos de Comentario y Adjuntos de Modo. Ambos hacen parte de la estructura del Modo. Los Adjuntos de Comentario expresan, como su nombre lo indica, un comentario acerca de la cláusula en general. Halliday (2014) da ejemplos de Adjuntos de Comentario como los siguientes: por supuesto, naturalmente, en verdad, se espera, tentativamente, desde el punto de vista (ético), etc. Por su parte, los Adjuntos de Modo expresan significados asociados a los sistemas de TIEMPO, INTENSIDAD y MODALIDAD marcados en el Finito. Halliday (2014) proporciona una lista de ítems que funcionan como Adjuntos de Modo, como los siguientes: de tiempo (en últimas, pronto, aún, todavía, ya, ya no, apenas, una vez que); de intensidad (totalmente, completamente, casi, escasamente, a duras penas, incluso, de hecho, en realidad, solo, simplemente); de modalidad, divididos en de habitualidad (siempre, nunca, ocasionalmente, rara vez) y, los que más interesan en este caso, de probabilidad (ciertamente, definitivamente, posiblemente, quizás, difícilmente, de ningún modo). Thompson (2014) hace ver que los Adjuntos de Modo "se sienten intuitivamente como más 'gramaticales' que los Adjuntos Circunstanciales", pero admite que, en algunos casos, "es difícil ver la diferencia" (p. 67).

Según Lavid et al. (2010), es difícil hacer un inventario de todas las formas de expresión de significados de una lengua, en particular porque a menudo se utilizan metáforas. En el caso de la probabilidad en español, mencionan como un ejemplo de expresión metafórica de la probabilidad el uso de procesos existenciales (haber, existir) o procesos relacionales intensivos de posesión (tener), unidos a otras palabras que expresan probabilidad:

Existe una alta probabilidad de que haya inundaciones también este año.

La representante de Colombia tiene una gran posibilidad de ganar medalla de oro.

Lavid et al. (2010) no lo señalan, pero los hablantes muy a menudo utilizamos marcaciones de probabilidad que no son congruentes con la realidad, ya poniéndolas demasiado cercanas a la Polaridad positiva, ya demasiado cercanas a la Polaridad negativa con fines expresivos o irónicos. Se puede agregar que, en estos casos, tanto el hablante como los interlocutores son conscientes de esta incongruencia con la realidad por cambios en la prosodia, en la gesticulación y hasta en la proxemia, por ejemplo:

Oh, sí, con toda seguridad que hoy mismo te traen el dinero. (Acompañado con una prosodia y una gestualidad dadas, de modo que tanto el hablante como el interlocutor saben que ello no es cierto, que no van a traer el dinero). 
Al aplicar estos conceptos al español, Lavid et al. (2010) presentan la probabilidad como un continuo de cuatro niveles que van de la máxima proximidad a la Polaridad positiva hasta la máxima proximidad al polo negativo. En esto se desvían de las propuestas de Halliday (2014) y Thompson (2014), para quienes bastan tres grados de probabilidad: Alto, Medio y Bajo. En esta exposición, se organizan las propuestas de Lavid et al. (2010) según la propuesta de Halliday (2014), como se muestra a continuación, aunque, como los lectores podrán verificar, no siempre todos los hablantes del español concordarán con ellas debido a diferentes variables sociolingüísticas, aparte de las expresiones metafóricas mencionadas.

El grado Alto en la escala de probabilidad, es decir, más cercano al polo positivo, lo da el verbo modal tener que (que en otros casos es utilizado para expresar también obligación) y la expresión más coloquial seguro que, derivada a su vez del proceso relacional es seguro que, como se ve en la Tabla 3. En el caso de tener que, el significado de probabilidad no se da con el infinitivo, como en tiene que llegar temprano.

Tabla 3. Marcación del grado Alto de la hipótesis mediante un Adjunto Modal

\begin{tabular}{|c|c|c|c|}
\hline $\begin{array}{l}\text { El profesor } \\
\text { Mi hijo }\end{array}$ & $\begin{array}{l}\text { ya tiene que } \\
\text { seguro que }\end{array}$ & $\begin{array}{l}\text { haber llegado, } \\
\text { no viene, }\end{array}$ & $\begin{array}{l}\text { porque su tren salía a las cinco. } \\
\text { aunque ya no está lloviendo muy } \\
\text { fuerte. }\end{array}$ \\
\hline Actor & $\begin{array}{c}\text { Adjunto } \\
\text { Modal } \\
\text { (probabilidad) }\end{array}$ & $\begin{array}{c}\text { Proceso: } \\
\text { Material }\end{array}$ & Circunstancia \\
\hline
\end{tabular}

Según los mismos autores, tener que, con Polaridad negativa, no puede aparecer para expresar este grado máximo de probabilidad. Esto crea una asimetría en el sistema modal del español, que es compensada con el uso de poder u otra construcción relacionada con posibilidad/probabilidad:

Mi hijo tiene que haber llegado ya.

Mi hijo no puede haber llegado todavía.

En cambio, seguro que puede aparecer tanto con Polaridad positiva como con negativa:

Seguro que le has contado todo.

Seguro que no le has contado todo.

Otras expresiones de grado Alto de probabilidad no mencionadas por estos autores son las que emplean léxico que acercan el enunciado a la Polaridad positiva, como en la Tabla 4: 
Tabla 4. Marcación del grado Alto de la hipótesis mediante léxico relacionado con probabilidad

\begin{tabular}{|l|l|}
\hline Creo que & el profesor está enfermo. \\
Mi creencia es que & nada grave va a pasar por la derogación de esa ley. \\
Presumo que & está resentido por mi actitud. \\
Se conjetura que & el funcionario se ha desaparecido para huir de deudas. \\
Según algunas hipótesis, & el lenguaje se originó en el canal viso-gestual. \\
Se supone que & no hay vida en Marte. \\
La suposición es que & hay complicidad de empleados en el robo. \\
\hline \multicolumn{1}{|c|}{ Adjunto } & \multicolumn{1}{c|}{ Proposición } \\
Modal & \\
\hline
\end{tabular}

El grado Medio de probabilidad viene dado por expresiones que tienen un significado de probabilidad similar: el modal deber de, que algunos hablantes utilizan también para la modulación de obligación o, viceversa, utilizan siempre el modal deber, que según la norma indica obligación"; los Adjuntos Modales seguramente, probablemente, en cuyo caso la probabilidad se disminuye si el Proceso principal está en Subjuntivo; el Proceso Relacional es probable que, que reduce a Portador el proceso principal y requiere el uso del Subjuntivo. La Tabla 5 presenta unos ejemplos:

Tabla 5. Marcación del grado Medio de la hipótesis mediante Adjunto Modal

Relacional

\begin{tabular}{|c|l|l|}
\hline El profesor no ha llegado, & $\begin{array}{l}\text { debe de } \\
\text { seguramente } \\
\text { es probable que } \\
\text { probablemente }\end{array}$ & $\begin{array}{l}\text { estar enfermo. } \\
\text { está enfermo. } \\
\text { esté enfermo. } \\
\text { no viene/venga hoy tampoco. }\end{array}$ \\
\hline Proposición & $\begin{array}{c}\text { Adjunto } \\
\text { Modal }\end{array}$ & Proposición \\
\hline
\end{tabular}

No mencionadas por Lavid et al. (2010), estarían en este mismo grado Medio expresiones como es como si, da la impresión de. Nótese una vez más cómo el uso del Subjuntivo disminuye el grado de probabilidad:

Es como si estuviera enfermo. (Probable)

Da la impresión de que está enfermo. (Probable)

Da la impresión de que estuviera enfermo. (Menos probable)

Por último, el grado Bajo de probabilidad se marca de diversas formas. Una de ellas es con el Futuro y el Condicional empleados con significado modal y no temporal, como se ve en la Tabla 6:

\footnotetext{
${ }^{4}$ Esta ambivalencia tiene muy posiblemente su origen en la desaparición, en la conversación cotidiana, del alófono fricativo de la oclusiva dental sonora /d/ y la consiguiente fusión de los dos fonos de la vocal media anterior /e/: debe de haber llegado > debe (d)e haber llegado > debe haber llegado.
} 
Tabla 6. Marcación del grado Bajo de la hipótesis mediante Futuro o Condicional con significado modal

\begin{tabular}{|c|l|c|l|l|}
\hline El profesor & $\begin{array}{l}\text { no ha llegado, } \\
\text { no vino } \\
\text { no ha venido }\end{array}$ & (el profesor) & $\begin{array}{l}\text { estará } \\
\text { estaría } \\
\text { habrá estado }\end{array}$ & enfermo. \\
\hline Actor & $\begin{array}{l}\text { Proceso: } \\
\text { Material }\end{array}$ & Portador & $\begin{array}{l}\text { Proceso: } \\
\text { Relacional } \\
\text { (atributivo) }\end{array}$ & Atributo \\
\hline
\end{tabular}

Otra marcación baja de probabilidad se da con los Adjuntos Modales tal vez, quizálquizás, posiblemente, a lo mejor, de pronto o capaz que, con el operador Finito modal puede que y los procesos relacionales atributivos es posible que, es factible que. Los dos primeros no afectan la construcción de la declarativa equivalente no marcada con probabilidad y aparecen a menudo en posición inicial en el enunciado. Así, prosiguiendo con el tema de los ejemplos anteriores, se pueden tener enunciados como los de la Tabla 7, con cambios en la conversación cotidiana en la prosodia, la gesticulación y la proxemia:

Tabla 7. Marcación del grado Bajo de la hipótesis mediante Adjuntos Modales, Operador Finito poder y Proceso Relacional (atributivo)

\begin{tabular}{|c|c|}
\hline $\begin{array}{l}\text { Tal vez } \\
\text { Quizá/Quizás } \\
\text { Puede que } \\
\text { Es posible/factible que }\end{array}$ & venga hoy \\
\hline $\begin{array}{c}\text { Adjunto } \\
\text { Modal }\end{array}$ & Proposición \\
\hline
\end{tabular}

Nótese que el último ejemplo indica también, además de probabilidad, la posibilidad o factibilidad de algo.

El grado más débil de probabilidad viene dado por el uso del Condicional de poder:

Según la directora, el profesor podría venir hoy.

La interpretación de todos los ejemplos anteriores, independientemente del grado de probabilidad, se complementa con la diferencia que Halliday (2014) hace ver entre "objetividad" y "subjetividad" en la marcación de la hipótesis. Esta se entrecruza, a su vez, con una dimensión "explícita-implícita", de modo que, aplicando los conceptos al español, yo creo es explícitamente subjetivo, es posible es explícitamente objetivo, puede ser es implícitamente subjetivo y quizás es implícitamente objetivo.

Ni Lavid et al. (2010) ni Halliday (2014) ni Thompson (2014) las incluyen como recursos para la marcación de la hipótesis en su tratamiento de la metafunción interpersonal, pero se pueden añadir aquí los complejos de cláusulas en los cuales hay por lo menos una subordinada condicional, ya afirmativa (si ..., siempre y cuando ...), ya negativa (a no ser que ..., a menos que ..). Lock (1996), sin embargo, sí las incluye en 
su lista de expresiones de probabilidad, aunque las restringe en inglés a la expresión de la predicción, mientras que en español pueden expresar también deducciones. La Tabla 8 muestra algunos ejemplos:

Tabla 8. Marcación de hipótesis mediante cláusulas condicionales de diferentes tipos

\begin{tabular}{|c|c|}
\hline Si me pagan, & vengo. (Condición real, predicción) \\
\hline Si me pagaran/pagasen, & $\begin{array}{l}\text { vengo. (Condición real, predicción con Subjuntivo que } \\
\text { reduce la probabilidad) }\end{array}$ \\
\hline Si no hubiera/hubiese habido paro, & yo habría llegado antes. (Condición irreal, predicción) \\
\hline Si no ha llegado el gerente, & es porque está ocupado. (Condición real, deducción) \\
\hline De estar enfermo, & $\begin{array}{l}\text { ya los otros empleados lo sabrían (Condición irreal } \\
\text { negativa, predicción) }\end{array}$ \\
\hline A no ser que no haya clases & $\begin{array}{l}\text { nos vemos en la universidad (Condición real negativa, } \\
\text { predicción) }\end{array}$ \\
\hline $\begin{array}{l}\text { Adjunto } \\
\text { Modal }\end{array}$ & Proposición \\
\hline
\end{tabular}

Por último, dado que, siguiendo a Lock (1996), se incluye la evidencialidad como un tipo de probabilidad, podemos considerar los Evidenciales, en español generalmente léxicos, como marcas de probabilidad. La Tabla 9 muestra ejemplos:

Tabla 9. Marcación de hipótesis mediante Evidencial léxico actuando como Adjunto Modal

\begin{tabular}{|c|c|}
\hline $\begin{array}{l}\text { Mi compañero dice que } \\
\text { Según dicen, } \\
\text { Es claro que }\end{array}$ & hoy no hay clases en la universidad. \\
\hline $\begin{array}{c}\text { Adjunto } \\
\text { Modal } \\
\text { (evidencial) }\end{array}$ & Proposición \\
\hline
\end{tabular}

Hay que resaltar entonces que en español y otras lenguas orales, tanto en la oralidad como en la escritura, hay una multitud de posibilidades. Como se ha visto, puede haber combinaciones de las anteriores, que marcan aún más que se trata de una hipótesis y no de un hecho establecido, sobre todo cuando van acompañadas en la oralidad de una prosodia, una gestualidad y hasta de una proxemia determinadas, que son más dicientes aun que las palabras acerca del grado de cercanía a uno u otro extremo de la Polaridad.

\section{La importancia de la gestualidad en la expresión de la hipótesis}

La hipótesis es una función que está íntimamente relacionada con la expresión de las emociones tanto con la cara como con el cuerpo. El trabajo de décadas de Ekman, quien se centró en la actividad de la cara y la cabeza, y que aparece resumido en Ekman (2003), le permitió constatar en efecto que, tal como lo había hecho ver un siglo antes Darwin (1998), la expresión de las emociones es universal en el género humano, contrario a lo que creían los antropólogos de la primera mitad del siglo XX, como 
Margaret Mead, Gregory Bateson, Edward Hall, Ray Birdwhistell y Charles Osgood. Una excepción era su mentor, Silvan Tomkins (1962), que no había podido probarlo. Ekman sí lo logró con sus investigaciones a lo largo y ancho del planeta. Un estudio más reciente (Sato et al., 2019) ha llegado a la misma conclusión. Sin embargo, aún queda por responder la pregunta de si debemos considerar la gestualidad, la pantomima y los emblemas que acompañan el habla como parte integral del enunciado (como se postula, por ejemplo, en Kendon, 1975, y en McNeill, 1992, 2005) o, incluso como hechos lingüísticos (como lo sugiere Hjelmslev, 1943/1971, ver más adelante).

Ekman y Friesen $(1975,1976)$ hicieron una presentación inicial de un procedimiento para describir y medir los diferentes movimientos faciales, que denominaron Facial Action Code y, más tarde, en Ekman y Friesen (1978), Facial Action Coding System o FACS, y que aparece más completamente ya en Ekman et al. (2002). El sistema, basado originalmente en una propuesta de Hjortsjö (1970), sirve para codificar los movimientos de la cara y la cabeza.

Si la expresión de las emociones es realmente universal, como parecen haber probado fehacientemente Ekman y sus colaboradores, la dificultad estriba entonces en dilucidar cuándo la praxis muscular es realmente lingüística o simplemente gestual. Tovar (2008) se vio abocado a resolver esta dificultad y, ante la falta de elementos para alcanzar la certidumbre, decidió acudir al concepto de "equifinalidad", propuesto por Feyereisen (1999) al estudiar los "gestos comunicativos". Según esta propuesta, los gestos, las palabras orales y las señas tienen el potencial de ser utilizados para expresar una misma intención comunicativa central, que sería en últimas lo que importa, algo que ya había anticipado Austin (1962). Sin embargo, el estado de los estudios en neuropsicología no ofrece todavía una respuesta concluyente.

Rinn (1991), en su tratamiento de la neuropsicología de la expresión facial, presenta, al igual que muchos otros autores, el inventario de los pares de nervios craneales, denominados por ello "pares craneales", que inervan cada uno de los dos lados de la cara, y cuyo inventario está establecido desde los estudios de von Sömmerring en el siglo XVIII (Li Ching et al., 2019), si bien por lo menos desde 1914 se ha identificado otro más, un "nervio terminal", "nervio nulla (n)", "par craneal 0" o "par craneal XIII", asociado con el órgano vomeronasal, aparentemente atrofiado en los humanos, pero que posiblemente tiene un papel en las funciones y los comportamientos reproductivos (Sonne y López-Ojeda, 2019). Las expresiones faciales se originan en patrones de contracción de los músculos faciales. La inervación motora de la cara viene principalmente del séptimo nervio craneal o par craneal VII, llamado por ello "nervio facial". Este par craneal VII emerge en la cara justo por debajo de la oreja y se subdivide en cinco ramas: temporal y zigomática, que inervan preferentemente la parte superior de la cara, y las ramas bucal, mandibular y cervical, que inervan preferentemente la parte inferior de la cara y el cuello, aunque se ha comprobado que hay variabilidad de un individuo a otro (De Bonnecaze et al., 2019; Raslan et al., 2017).

Algunos otros aspectos de la expresión facial y de la lengua son controlados por otros pares craneales. Así, el par craneal III u "óculo-motor" inerva los músculos que abren y cierran los ojos y gobierna el diámetro de la pupila; los pares craneales III, IV y VI 
rigen los movimientos del globo ocular; finalmente, el par craneal V o "trigémino" inerva a su vez los músculos de la mandíbula (Rinn, 1991). Se agregan aquí, de Marieb (2008), otros no mencionados por Rinn (1991), pero que tienen que ver con la expresión en señas: el par craneal IX o "glosofaríngeo", el par craneal X o "vago", el par craneal XI o "espinal" y el par craneal XII o "hipogloso mayor", que coordinan, además de otros movimientos, los de la lengua, la laringe, la faringe, el cuello y los hombros. El par craneal VIII o "vestíbulo-coclear", por su parte, inerva los órganos de la audición, así como los del equilibrio, por lo que sirve para la comunicación en ambas modalidades.

Sin embargo, no hay aún claridad acerca de los circuitos neuronales que originan las contracciones musculares faciales ni el papel de los diferentes componentes del sistema límbico. Sí se ha determinado, en cambio, como lo hace ver Gothard (2014), que los movimientos faciales pueden ser voluntarios (coordinados por vías corticales), reflejos o involuntarios (coordinados por vías motoras subcorticales, ubicadas principalmente en el tronco encefálico). Según esta misma autora, las áreas subcorticales muy difícilmente pueden dirigir una expresión facial hacia un individuo de interés, como se requiere durante las interacciones sociales no ritualizadas. Rinn (1991) sostiene que, en general, los músculos inervados exclusivamente por el córtex contralateral, como es el caso primordialmente de las manos y la cara, tienen el grado más alto de motricidad fina y tienden a participar en los movimientos inducidos voluntariamente, lo que es particularmente cierto en el caso de los músculos de la parte inferior de la cara y la lengua. Por contraste, los músculos de la parte superior de la cara tienen un mayor porcentaje de inervación ipsilateral, lo que hace que participen menos en los movimientos voluntarios.

244 Los movimientos voluntarios son entonces corticales y tienden a ser respuestas aprendidas; los involuntarios o de respuesta son subcorticales y son innatos. Esto implicaría que, aunque el tema no se ha estudiado en profundidad ni desde la lingüística de señas ni desde la neuropsicología, en lenguas de señas, los movimientos de los músculos de la parte inferior de la cara (mejillas, labios, lengua, barbilla) que expresan hipótesis podrían caracterizarse inequívocamente como lingüísticos, mientras que los movimientos de la parte superior de la cara (nariz, ojos, párpados y ceño) serían en gran parte innatos y, posiblemente, comunes a los de hablantes de otras lenguas de señas y de lenguas orales. Pero dado que pueden ser en ocasiones voluntarios, en muchos casos ya harían parte de la léxico-gramática de la lengua de señas en cuestión, como ya apuntaba Wilbur (1991), cuando señalaba la utilización distintiva de las cejas en la determinación de todas las cláusulas no declarativas en la lengua de señas norteamericana (ASL).

No obstante, la determinación de su carácter no es tan fácil. Ekman y Friesen (1975) encontraron que los movimientos de la cara en los oyentes están sujetos a las denominadas display rules o reglas acerca de lo que diferentes individuos en las diferentes culturas han aprendido a mostrar o suprimir, lo que puede estar en el origen de la creencia de que los gestos faciales cambiaban según la cultura. Y aunque Ekman (2003) asegura que el movimiento innato para expresar una emoción alcanza a observarse durante fracciones de segundos, en la práctica esto hace suponer que ciertos movimientos musculares que se dan espontáneamente en la marcación de la hipótesis, tanto en lenguas de señas como orales, pueden ser obviados en algunos casos por los señantes y hablantes, lo que complica la determinación de la naturaleza, lingüística o innata, de la marcación de esta función. 
Tal vez a esto se refieren también Sato et al. (2019) cuando presentan los resultados de varios estudios, según los cuales no siempre las emociones se expresan con movimientos faciales prototípicos. Según ellos, para perfeccionar la teoría universalista, se requiere de más investigaciones sobre la expresión facial en las dimensiones afectivas y en los mensajes sociales. Por ello, si bien en discurso elicitado es factible, como se verá, reconocer ciertos rasgos no manuales como marcadores de la hipótesis, estos no siempre aparecerán en el discurso espontáneo en señas, tal como sucede en el discurso oral. Y, cuando no aparezcan, quedará siempre la duda de si fue por una regla social, dependiente de la situación de comunicación, o por otra idiosincrasia, o si influye en esto el que estén presentes o no en el enunciado palabras o señas que inequívocamente expresan hipótesis.

\section{Metodología}

La metodología es muy similar a la seguida en Tovar (2017), para el estudio de la definición y en Tovar y López (2018), para la clasificación en LSC. A continuación, aparecen los detalles.

\section{Informantes}

Los informantes fueron tres maestros de una escuela para niños sordos de Cali, usuarios de la LSC, de entre 28 y 34 años. Según el diagnóstico de la audiometría, el informante $\mathrm{A}$ y la informante $\mathrm{C}$ son sordos profundos bilaterales, pero el informante $B$ no recuerda el resultado del suyo, solo sabe que nunca pudo con la terapia oral con audífono, que resistió solo dos meses. Todos reportan algunos restos auditivos en uno o ambos oídos, y todos tienen historia de educación oralista en mayor o menor grado. Uno de ellos, el informante $B$, posee título de tecnólogo y está a punto de terminar estudios de nivel de profesional universitario; los otros dos tienen título de tecnólogo. El co-autor del artículo, intérprete de profesión, ayudó en la comprensión por los sordos de partes difíciles de los textos escritos que se utilizaron de base.

\section{Recopilación, transcripción y análisis del corpus}

Una vez más, se hizo un trabajo de discusión metalingüística con los tres informantes sordos a partir de textos de hipótesis en español escrito, con aclaraciones de los autores cuando era necesario, ya que las disciplinas se estructuran como lenguajes (Greimas, 1970/1973, Halliday 2004), y es conveniente encontrar formas de expresión paralelas en la comunicación cara a cara en lengua de señas. En efecto, el lenguaje académico ya es un "hecho social", definido por Durkheim (1895/1982) como

toda manera de hacer, fija o no, susceptible de ejercer sobre el individuo una coacción exterior; o también, que es general dentro de la extensión de una sociedad dada a la vez que tiene una existencia propia, independiente de sus manifestaciones individuales. (p. 46). 
El corpus se recopiló durante seis sesiones de tres horas cada una, con un total de 33 textos seleccionados, que fueron producidos y memorizados por los informantes sordos, 25 de ellos de hipótesis de la vida diaria, de 15 segundos de duración en promedio, y 8 de hipótesis científica, de 35 segundos de duración en promedio, transcritos por los dos autores y analizados por el autor principal. De hecho, esta vez el trabajo se inició con la elicitación de enunciados de hipótesis de la vida diaria, con el fin de determinar el papel importante de los rasgos no manuales en la expresión de esta función comunicativa en la LSC, que se notó desde un trabajo preparatorio. Por ello, durante la transcripción y el análisis, aparte de continuar utilizando el marco teórico de Liddell y Johnson (1989), Liddell (2003), Oviedo (2001, 2004), complementado con las actualizaciones de Johnson (2009, 2010a, 2010b), hubo que prestar una mayor atención a los rasgos no manuales. Para ello, se tuvieron en cuenta las propuestas descriptivas de los movimientos faciales de Ekman (2003) y Ekman et al. (2002), y se utilizó para su interpretación la información básica sobre la neuropsicología de la praxis muscular facial, como aparece en el marco teórico, confirmando con los informantes en algunos casos en que había dudas.

El protocolo principal de análisis fue, entonces, la transcripción de las grabaciones en video mediante una matriz con glosas de señas completamente especificadas léxicamente, dadas inicialmente por el intérprete co-autor y discutidas en algunos casos, y transcripciones fonéticas de las no especificadas, hechas por el investigador principal. Se transcribieron también los rasgos no manuales que hacen marcaciones léxico-gramaticales, haciendo énfasis en los que tienen que ver con la hipótesis. Tanto las glosas como los rasgos no manuales identificados como marcadores de la hipótesis aparecen en cursivas. Las matrices se acompañaron de sus respectivas traducciones libres, siguiendo la experiencia del co-autor intérprete.

Otras convenciones de transcripción fueron las siguientes: En el caso de los gestos que tienen una marcación léxica o gramatical inequívoca, esta aparece entre paréntesis cuadrados [ ]; las glosas separadas por guiones (-) se refieren a una seña que equivale a más de una palabra en la glosa en español; las letras separadas por guiones se refieren a digitalizaciones; y los compuestos aparecen marcados uniendo cada elemento por un acento circunflejo $\left({ }^{\wedge}\right)$, como lo propone el modelo de Halliday $(2004,2014)$ y no con una barra (/), como hacen otros autores de señas, lo que puede llevar a confusiones, pues el mismo signo se utiliza también para expresar un significado alternativo. Se incluyó esta vez información sobre el grado de intensidad de los articuladores no manuales, que Ekman et al. (2002) caracterizan en una escala de cinco niveles: A (rastros), B (leve), C (marcado o pronunciado), D (severo o extremo) y E (máximo). Para efectos prácticos, los retradujimos en una escala desde 1 (rastros) hasta 5 (máximo) y, con base en el trabajo de elicitación inicial hecho con los informantes, identificamos cuándo eran realmente pertinentes para la expresión de la hipótesis e intentamos determinar cuándo podían considerarse lingüísticos y cuándo eran gestualidad en común con los oyentes. Se prefirió también seguir utilizando el inventario de rasgos no manuales identificados en la literatura de lingüística de señas, en lugar de la identificación mediante números de las diferentes unidades de acción 
(action units) de la cara y la cabeza propuestas en el FACS. Así, por ejemplo, se ha preferido conservar el rasgo de NarizFruncida, siguiendo a Liddell y Johnson (1989), en lugar del número de la unidad de acción "AU 9" de Ekman et al. (2002), que se refiere a la contracción del músculo levator labii superioris alaeque nasi, o el de CeñoFruncido, en lugar de "AU 4", que incluye la acción de los músculos depressor glabellae, depressor supercilii y corrugator supercilii. En el proceso, pudimos identificar algunos rasgos no manuales no identificados en la literatura conocida, tanto faciales (por ejemplo, LabioExplota o LabioInhala) como corporales (por ejemplo, TorsoArqueado o FlexiónRodilla), que tienen significado en el enunciado y no parecen ser pantomimas.

Finalmente, para el análisis léxico y gramatical, se utilizó el modelo de Gramática Sistémico-Funcional de Halliday (2014), complementado con Thompson (2014) y Lock (1996), con los ajustes necesarios a la modalidad viso-gestual. En las tablas de transcripción, aparecen tres filas: la primera, para los rasgos no manuales (RNM) distintivos, es decir los movimientos del cuerpo, la cabeza, el ceño, los párpados, el contacto visual $(\mathrm{C}+)$, la dirección de la mirada, la nariz, la mejilla, la boca, la lengua y la mandíbula (cuando se dan cambios en un RNM que acompaña una seña manual, se utiliza una flecha $\rightarrow$ ); la segunda, para la mano activa o dominante (MA); y la tercera, para la mano pasiva, subordinada o no dominante (MP). El símbolo indica parpadeo, que podría marcar límite de cláusula u otra unidad sintagmática, o ser simplemente un recurso fisiológico para el humedecimiento de la córnea (Baker y Padden, 1978; Tovar, 2006; Wilbur, 1994).

\section{HALLAZGOS SOBRE LA HIPÓTESIS EN LSC}

En la LSC se encontró que la hipótesis también se marca con grados de probabilidad, desde la absoluta certeza hasta la más vaga probabilidad/posibilidad. Tal como se ve en español, hay marcaciones léxicas. Pero hay también marcaciones importantes con rasgos no manuales. Los resultados de la elicitación se hicieron a partir de la cláusula declarativa sin modalidad que se muestra en la Figura 1. A estos se añadieron otros casos encontrados en los textos de hipótesis académica analizados: 

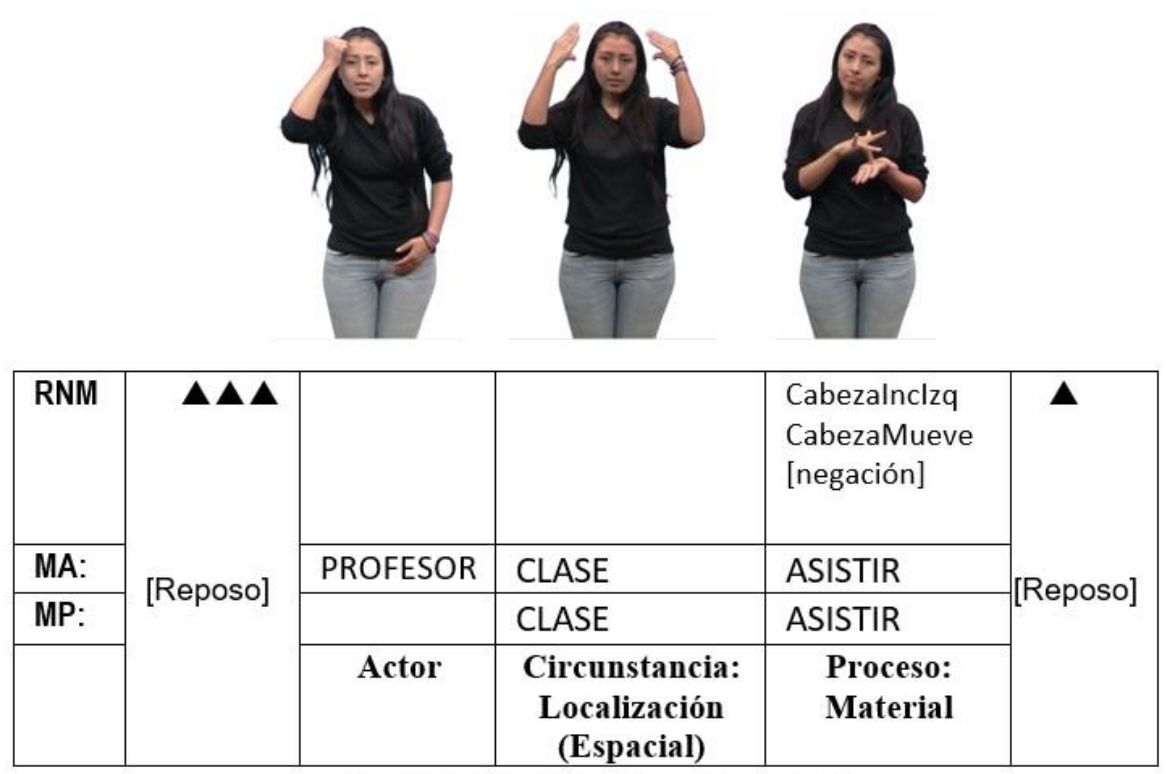

|| el profesor no vino a clase $\|$

Figura 1. Toma 4: Declarativa sin modalidad (Informante C)

Una vez se tuvo la elicitación a partir de un corpus basado en la expresión de la hipótesis en español, se procedió a establecer una gradación de las posibilidades desde la máxima Polaridad positiva hasta puntos más cercanos a la Polaridad negativa. Se encontró que en la LSC, debido a la multiplicidad de articuladores manuales y no manuales, y a sus diferentes combinaciones posibles, más las señas léxicas que indican hipótesis, es aparentemente posible establecer una mayor gradación que en inglés o en español, lo que causa dificultad al momento de decidir cómo traducir los enunciados 5 . Sin embargo, para efectos de conservar el modelo propuesto por Halliday (2014) y poder establecer comparaciones con las lenguas orales, se decidió aquí también establecer tres niveles de probabilidad. En esta determinación participaron los informantes A y C. Los resultados son como sigue, aunque, tal como sucede en el caso de las lenguas orales, posiblemente no todos los señantes estarán siempre de acuerdo. Las marcaciones de hipótesis aparecen en letras bastardillas.

\section{Nivel Alto de probabilidad}

El nivel Alto de probabilidad se marca léxicamente mediante Adjuntos Modales de probabilidad o Procesos verbales o mentales (por ejemplo, CREER, SEGURO o

\footnotetext{
${ }^{5}$ En realidad, esto es solo parcialmente cierto, ya que, como se ha visto, también en la lengua oral sería factible establecer una gradación más fina mediante la combinación de los elementos sintagmáticos con la prosodia y la gestualidad. Es el tipo de situaciones que le da la razón a Hjelmslev (1943/1971) cuando afirma, reconociendo el aporte de Zwirner y Zwirner (1937), que "es erróneo suponer que la sustancia de la expresión de un lenguaje [sic] hablado consta exclusivamente de sonidos, ya que el habla puede ir acompañada del gesto y ciertos componentes del habla reemplazarse por el gesto y que en realidad, no solamente los llamados órganos de la articulación (garganta, boca y nariz), sino casi toda la musculatura estriada coopera en la práctica del lenguaje 'natural'" (pp. 146-147).
} 
POSIBLE) que expresan inequívocamente la probabilidad, y que van acompañados regularmente de rasgos no manuales típicos, sobre todo de expresión facial e inclinación y ladeo de la cabeza o del cuerpo. Según su significado, estos Adjuntos Modales léxicos, con sus respectivos rasgos no manuales, indican nivel alto de probabilidad. Los Adjuntos Modales léxicos aparecen casi siempre en el último lugar, tal como sucede a veces en la oralidad. Las Figuras 2, 3, 4, 5 y 6 muestran estos niveles altos del mayor al menor:

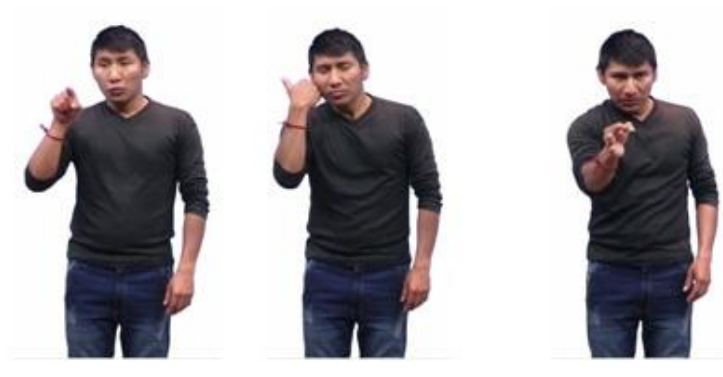

\begin{tabular}{|c|c|c|c|c|c|}
\hline RNM & \multirow{5}{*}{ [Reposo] } & $\begin{array}{l}\text { g[u:] } \\
\text { [i.e. 'tú', } \\
\text { marca } \\
\text { idiosincrásica } \\
\text { de pronombre] }\end{array}$ & $\begin{array}{l}\text { CabezalncDer } 2 \\
\text { CabezaAdelante } 2 \\
\text { CeñoFruncido } 2 \\
\text { LabioApretado2 }\end{array}$ & $\begin{array}{l}\text { Cuerpolncl } \\
\text { Adelante } 2 \\
\text { CabezaAdelante2 } \\
\mathrm{C}+\end{array}$ & \multirow{5}{*}{ [Reposo] } \\
\hline MA: & & PRO-3sg & ENFERMO & SEGURO & \\
\hline \multirow[t]{3}{*}{ MP: } & & & & & \\
\hline & & $\begin{array}{l}\text { Portador: } \\
\text { Poseedor }\end{array}$ & $\begin{array}{c}\text { Proceso: Rel. } \\
\text { Intensivo } \\
\text { (Atribución) }\end{array}$ & \multirow[t]{2}{*}{$\begin{array}{c}\text { Adjunto } \\
\text { Modal } \\
\text { (Probabilidad) }\end{array}$} & \\
\hline & & & Atributo & & \\
\hline
\end{tabular}

Figura 2. Toma 10: Hipótesis marcada mediante Adjunto Modal equivalente a "es seguro" con rasgos no manuales de Cuerpo y Cabeza inclinado hacia adelante (Informante B) 

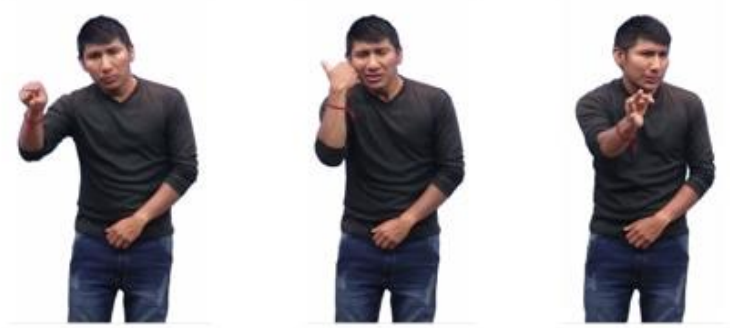

\begin{tabular}{|c|c|c|c|c|c|}
\hline RNM & \multirow{5}{*}{ [Reposo] } & & $\begin{array}{l}\text { CabezaAdelante } 1 \\
\text { CabezalnclDer } \\
\mathrm{C}+\end{array}$ & $\begin{array}{l}\text { CabLadlzq } \\
\text { OjoSemicerrado } \\
\text { ['seguro'] } \\
\text { C+ }\end{array}$ & \multirow{5}{*}{ [Reposo] } \\
\hline MA: & & $\begin{array}{l}\text { PRO-3sg } \\
\text { [pV2pecho] }\end{array}$ & ENFERMO & $\begin{array}{l}\text { SEGURO } \\
\text { [con fortaleza y } \\
\text { detención súbita] }\end{array}$ & \\
\hline \multirow[t]{3}{*}{ MP: } & & & & & \\
\hline & & \multirow[t]{2}{*}{$\begin{array}{l}\text { Portador: } \\
\text { Poseedor }\end{array}$} & $\begin{array}{c}\text { Proceso: Rel. } \\
\text { Intensivo } \\
\text { (Atribución) }\end{array}$ & \multirow[t]{2}{*}{$\begin{array}{c}\text { Adjunto } \\
\text { Modal } \\
\text { (Probabilidad) }\end{array}$} & \\
\hline & & & Atributo & & \\
\hline
\end{tabular}

|| él está enfermo, seguro \|

[= él seguro que está enfermo]

Figura 3. Toma 13B: Hipótesis marcada mediante Adjunto Modal a "seguro que" y rasgos no manuales de Ojos semicerrados y Cabeza ladeada (Informante B)
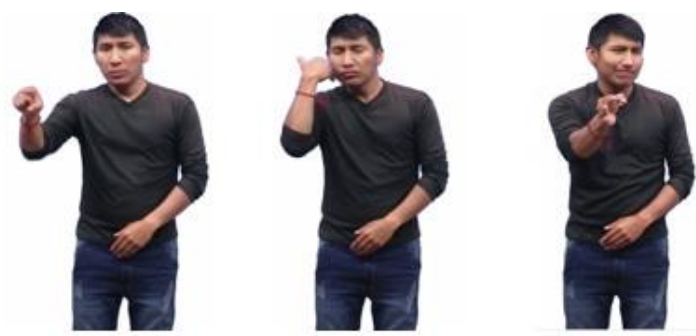

\begin{tabular}{|c|c|c|c|c|c|}
\hline RNM & \multirow[t]{5}{*}{ [Reposo] } & $\begin{array}{l}\text { CabezalncDer } \\
\text { CabLadDer1 } \\
\mathrm{C}+ \\
\text { g[u:] } \\
\text { [i.e. 'tú', marca } \\
\text { idiosincrásica } \\
\text { de pronombre] }\end{array}$ & & $\begin{array}{l}\text { CuerpoAdelante } \\
\text { CabezaLadlzq } \\
\text { CabezaAsiente } \\
\text { [i.e. 'es seguro'] } \\
\text { CeñoFruncido } 3\end{array}$ & \multirow{5}{*}{ [Reposo] } \\
\hline MA: & & $\begin{array}{l}\text { PRO-3sg } \\
\text { [pV2cara] }\end{array}$ & ENFERMO & SEGURO & \\
\hline \multirow[t]{3}{*}{ MP: } & & & ENFERMO & & \\
\hline & & $\begin{array}{l}\text { Portador: } \\
\text { Poseedor }\end{array}$ & $\begin{array}{c}\text { Proceso: Rel. } \\
\text { Intensivo } \\
\text { (Atribución) }\end{array}$ & \multirow[t]{2}{*}{$\begin{array}{c}\text { Adjunto } \\
\text { Modal } \\
\text { (Probabilidad) }\end{array}$} & \\
\hline & & & Atributo & & \\
\hline
\end{tabular}

Figura 4. Toma 11A: Hipótesis marcada mediante Adjunto Modal equivalente a "es seguro que" con rasgos no manuales de Cuerpo inclinado adelante y Cabeza ladeada asintiendo con Ceño fruncido (Informante B) 


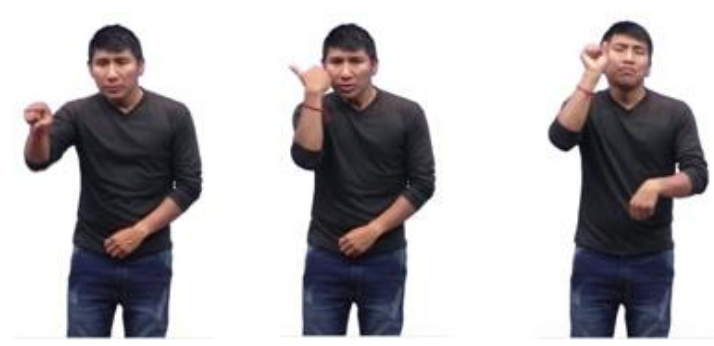

\begin{tabular}{|c|c|c|c|c|c|}
\hline RNM & \multirow{5}{*}{ [Reposo] } & & $\begin{array}{l}\text { CabezaAdelante } 1 \\
\text { CabezalnclDer } \\
\text { C+ }\end{array}$ & $\begin{array}{l}\text { CabezaAtrás } \\
\text { CabLadlzq } 2 \\
\rightarrow \text { CabLadlzq } 3 \\
\text { C+ } \\
\text { LabioApretado } 1\end{array}$ & $\Delta$ \\
\hline MA: & & $\begin{array}{l}\text { PRO-3sg } \\
\text { [pV2pecho] }\end{array}$ & ENFERMO & POSIBLE & \multirow[t]{4}{*}{ [Reposo] } \\
\hline \multirow[t]{3}{*}{ MP: } & & & & POSIBLE & \\
\hline & & $\begin{array}{l}\text { Portador: } \\
\text { Poseedor }\end{array}$ & $\begin{array}{l}\text { Proceso: Rel. } \\
\text { Intensivo } \\
\text { (Atribución) }\end{array}$ & \multirow[t]{2}{*}{$\begin{array}{c}\text { Adjunto } \\
\text { Modal } \\
\text { (Probabilidad) }\end{array}$} & \\
\hline & & & Atributo & & \\
\hline
\end{tabular}

Figura 5. Toma 13A: Hipótesis marcada mediante Adjunto Modal equivalente a "es posible que" con rasgos no manuales de Cabeza inclinada atrás y ladeada, con Labios apretados (Informante B)

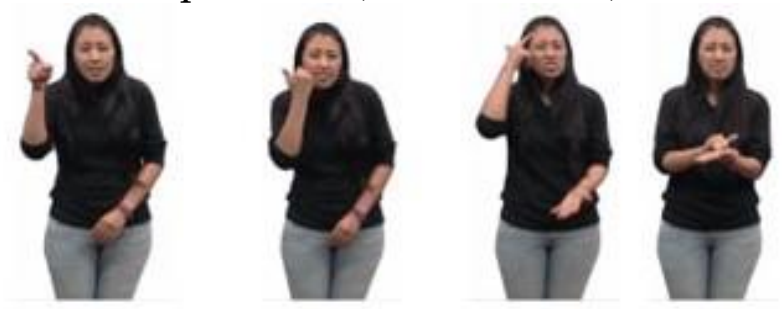

\begin{tabular}{|c|c|c|c|c|c|}
\hline RNM & \multirow[t]{5}{*}{ [Reposo] } & $\begin{array}{l}\text { CabezalncDer } 1 \\
\text { CabezaAdelante } \\
\text { HombroArriba } \\
\text { MiradaDerecha }\end{array}$ & $\begin{array}{l}\text { CejaArqueada } \\
\text { OjoSemicerrado } \\
\mathrm{C}+ \\
\text { [eisi.e. 'enfermo'] }\end{array}$ & $\begin{array}{l}\text { CabezaNeutra } \\
\rightarrow \text { CabezaAsiente } \\
\text { HombroNeutro } \\
\text { CeñoFruncido } \\
\text { OjoSemicerrado } 3 \\
\text { C+ } \\
\text { NarizFruncida } \\
\text { LabioNeutro }\end{array}$ & \multirow[t]{5}{*}{ [Reposo] } \\
\hline MA: & & $\begin{array}{l}\text { PRO-3sg } \\
\text { [pV2 cara] x2 }\end{array}$ & ENFERMO & CREER & \\
\hline \multirow[t]{3}{*}{ MP: } & & & & CREER & \\
\hline & & $\begin{array}{l}\text { Portador: } \\
\text { Poseedor }\end{array}$ & $\begin{array}{c}\text { Proceso: Rel. } \\
\text { Intensivo } \\
\text { (Atribución) }\end{array}$ & \multirow[t]{2}{*}{$\begin{array}{c}\text { Adjunto } \\
\text { Modal } \\
\text { (Probabilidad) }\end{array}$} & \\
\hline & & & Atributo & & \\
\hline
\end{tabular}

Figura 6. Toma 8: Hipótesis marcada mediante Proceso Mental CREER como Adjunto Modal y rasgos no manuales de Cabeza asintiendo, Ceño y Nariz fruncidos y Ojos semicerrados (Infomante C) 


\section{Nivel Medio de probabilidad}

El nivel Medio de probabilidad se marca primordialmente con Adjuntos Modales, que pueden ser procesos mentales de marcación de hipótesis, como PARECER o RAZONAR/PREGUNTARSE, $u$ otros recursos léxicos, como SEGURO, POSIBLE, que comportan sus propios rasgos no manuales. Asimismo, puede haber rasgos no manuales, solos o combinados con otras señas, sobre lo cual no se puede generalizar, porque es idiosincrásico. También se usan el Finito modal PODER, una marcación de EVIDENCIALIDAD (DECIR) o gestos unimanuales o bimanuales (1^/o- c+ o 1234+sep/a+), equivalentes a un Adjunto Modal (probabilidad). El primero, gesto unimanual 1^/o$\mathrm{c}^{+}$, con contacto de la yema distal en la sien y acompañado por los rasgos de LabioEntreabierto y LenguaProtruida, indicando incertidumbre y a menudo interpretado como PENSAR, CREER, SUPONER u otros Procesos Mentales similares en pretérito indefinido o imperfecto, ha llevado también a la hipótesis de que sería la única marca de TIEMPO verbal primario en LSC, pero probablemente indica más bien suposición. El gesto 1234+sep/a+, unimanual o bimanual, con diferentes orientaciones de la palma y rotaciones de antebrazo, es denominado crecientemente en la literatura de señas como PALM-UP. Se lo utiliza universalmente tanto en la oralidad como en las diferentes lenguas de señas y en los sistemas de señas hogareñas con el significado central de "ausencia de conocimiento" y sus ramificaciones, incluyendo incertidumbre e hipótesis (Cooperrider et al., 2018). Ambos posiblemente ya estén volviéndose parte de la léxico-gramática de la LSC, como señalan estas autoras para otras lenguas de señas, pero ante la falta de acuerdo entre los señantes, los presentamos aquí todavía como gestos con función de diferentes tipos de Adjuntos Modales. Una vez más se ve cómo constantemente los Adjuntos Modales, léxicos o gestuales, aparecen siempre en posición final, como se ve en las Figuras 7, 8, 9, 10, 11, 12, 13, 14, 15, 16 y 17, de nuevo organizadas del nivel Medio más alto al nivel Medio más bajo, según indican los informantes: 


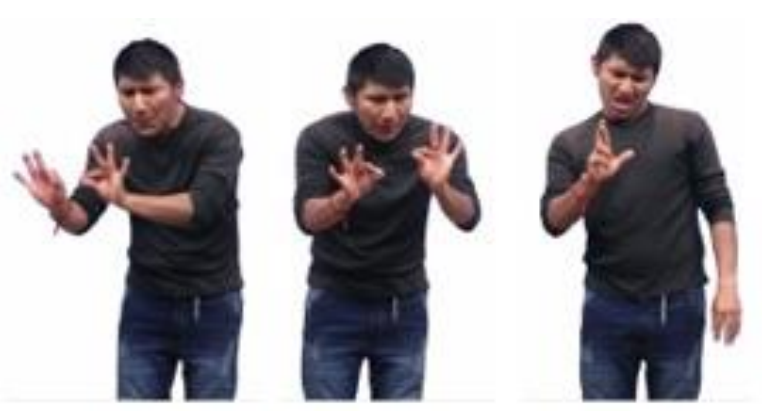

\begin{tabular}{|c|c|c|c|}
\hline RNM & \multirow{5}{*}{ [Reposo] } & $\begin{array}{l}\text { CuerplncAdelant } \\
\text { CeñoFruncido } \\
\text { LabioRetraído } \\
\text { LabioApretadoe }\end{array}$ & $\begin{array}{l}\text { CuerpoNeutro } \\
\text { TroncoArqueado } \\
\text { CabezaLadeadalzq } \\
\text { OjoSemicerrado } 4 \\
\text { MiradaAbajolzq }\end{array}$ \\
\hline MA: & & $\begin{array}{l}\text { NADIE-DICE- } \\
\text { NADA }\end{array}$ & RARO \\
\hline \multirow[t]{3}{*}{ MP: } & & $\begin{array}{l}\text { NADIE-DICE- } \\
\text { NADA }\end{array}$ & \\
\hline & & $\begin{array}{l}\text { Portador: } \\
\text { Poseedor }\end{array}$ & $\begin{array}{c}\text { Proceso: Rel. } \\
\text { Intensivo } \\
\text { (Atribución) }\end{array}$ \\
\hline & & & Atributo \\
\hline
\end{tabular}
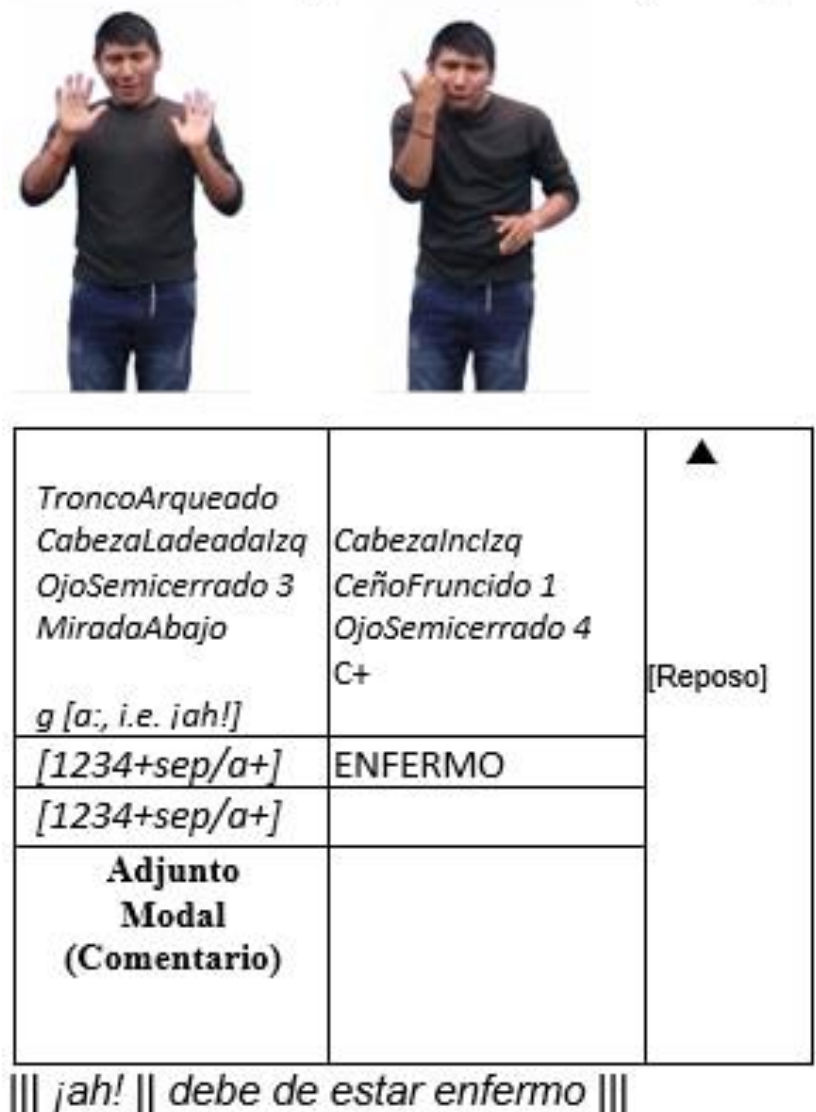

Figura 7. Toma 16A: Hipótesis marcada mediante gesto bimanual con rasgos no manuales de Tronco arqueado, Cabeza ladeada, Ojos semicerrados y Mirada abajo, todo equivalente a "deber de" (Informante B) 


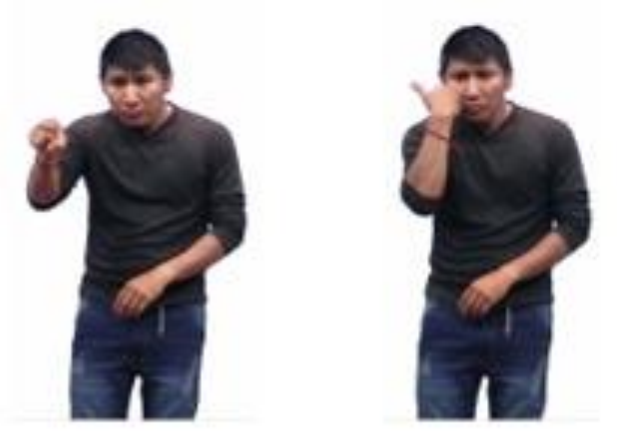

\begin{tabular}{|c|c|c|c|}
\hline RNM & \multirow{5}{*}{ [Reposo] } & $\begin{array}{l}\text { CabezaAdelante } \\
\text { MiradaDerecha }\end{array}$ & $\begin{array}{l}\text { CabezaAsiente } \\
\text { OjoSemicerrado } \\
\text { MiradaAbajo } \\
\text { CeñoFruncido } 4 \\
\text { LabioRetraído }\end{array}$ \\
\hline MA: & & PRO-3sg & ENFERMO \\
\hline \multirow[t]{3}{*}{ MP: } & & & \\
\hline & & \multirow[t]{2}{*}{$\begin{array}{l}\text { Portador: } \\
\text { Poseedor }\end{array}$} & $\begin{array}{c}\text { Proceso: Rel. } \\
\text { Intensivo } \\
\text { (Atribución) }\end{array}$ \\
\hline & & & Atributo \\
\hline
\end{tabular}

II está enfermo,
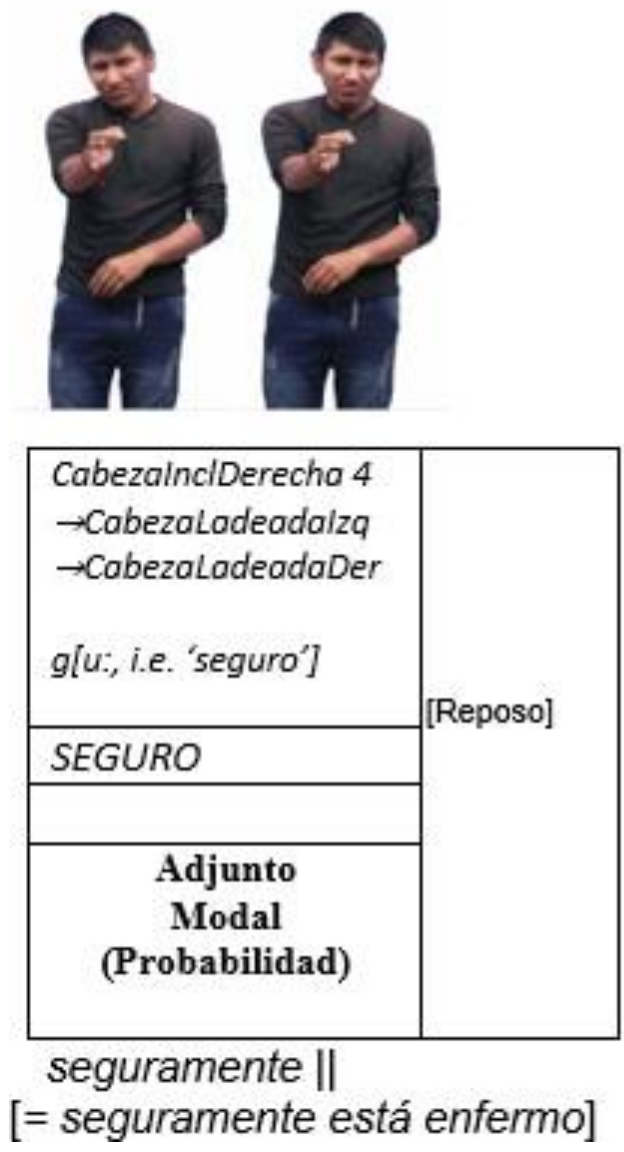

Figura 8. Toma 16B: Hipótesis marcada mediante seña léxica SEGURO como

Adjunto Modal, con rasgos no manuales de Cabeza inclinada y ladeo a ambos lados (Informante B) 


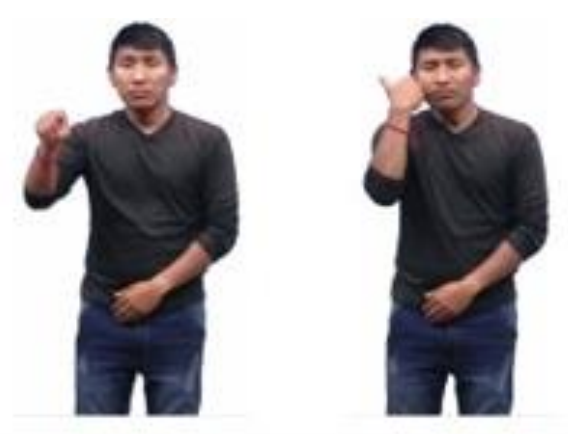

\begin{tabular}{|c|c|c|c|}
\hline RNM & \multirow{5}{*}{ [Reposo] } & & $\begin{array}{l}\text { CabezaLadeadaDer } \\
\text { LabioRetraído } \\
\text { LabioEntreabierto } 3\end{array}$ \\
\hline MA: & & $\begin{array}{l}\text { PRO-3sg } \\
\text { [pV2cara] }\end{array}$ & ENFERMO \\
\hline \multirow[t]{3}{*}{ MP: } & & & \\
\hline & & \multirow[t]{2}{*}{$\begin{array}{l}\text { Portador: } \\
\text { Poseedor }\end{array}$} & $\begin{array}{l}\text { Proceso: Rel. } \\
\text { Intensivo } \\
\text { (Atribución) }\end{array}$ \\
\hline & & & Atributo \\
\hline
\end{tabular}

\section{|| está enfermo}
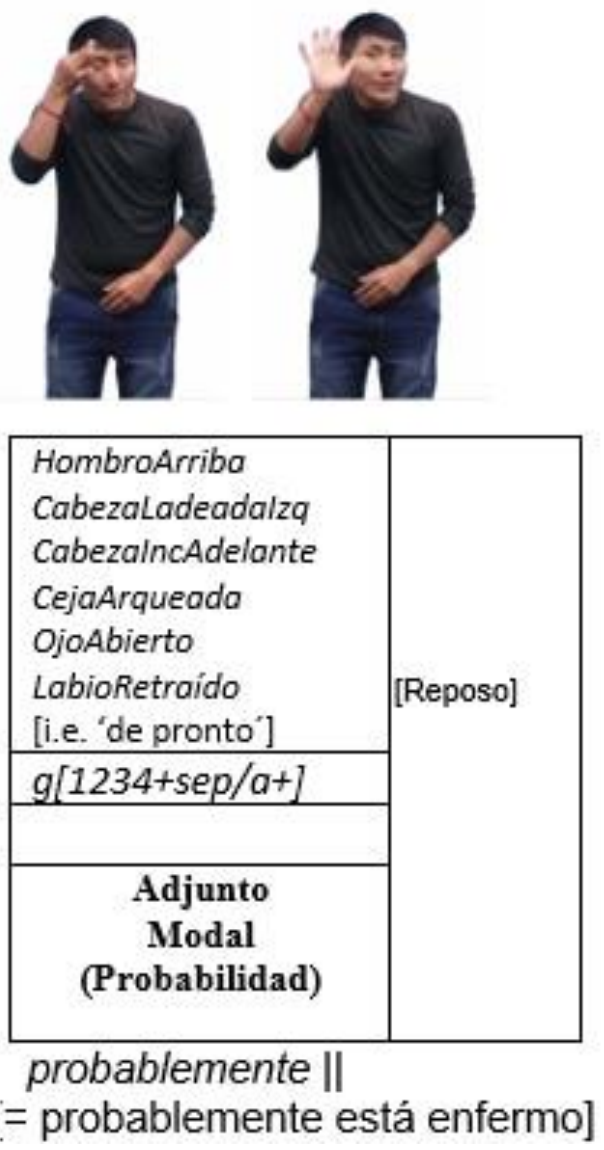

Figura 9. Toma 11C: Hipótesis marcada con gesto unimanual como Adjunto Modal, acompañado con rasgos no manuales de Hombros arriba, Cabeza inclinada hacia adelante y ladeada, Cejas arqueadas, Ojos bien abiertos y Labios retraídos (Informante B) 


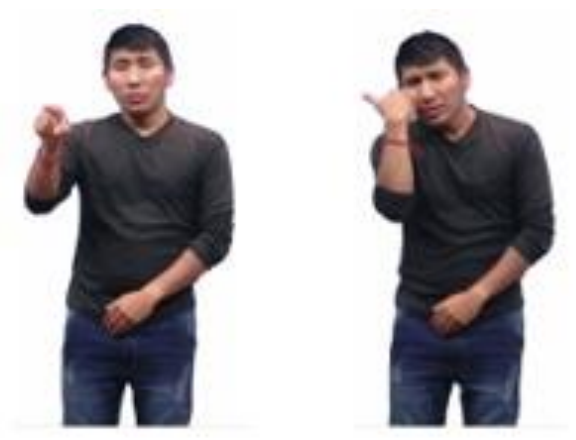

\begin{tabular}{|c|c|c|c|}
\hline RNM & \multirow{5}{*}{ [Reposo] } & $\begin{array}{l}\text { CabezaLadDer } 2 \\
\text { OjoSemicerrado } 2 \\
\text { [i.e., 'enfermo'] }\end{array}$ & $\begin{array}{l}\text { CabezaLad Der } 3 \\
\text { OjoSemicerrado } 3 \\
\text { [i.e., 'enfermo'] }\end{array}$ \\
\hline MA: & & $\begin{array}{l}\text { PRO-3sg } \\
\text { [pV2cara] }\end{array}$ & ENFERMO \\
\hline \multirow[t]{3}{*}{ MP: } & & & \\
\hline & & \multirow[t]{2}{*}{$\begin{array}{l}\text { Portador: } \\
\text { Poseedor }\end{array}$} & $\begin{array}{c}\text { Proceso: Rel. } \\
\text { Intensivo } \\
\text { (Atribución) }\end{array}$ \\
\hline & & & Atributo \\
\hline
\end{tabular}

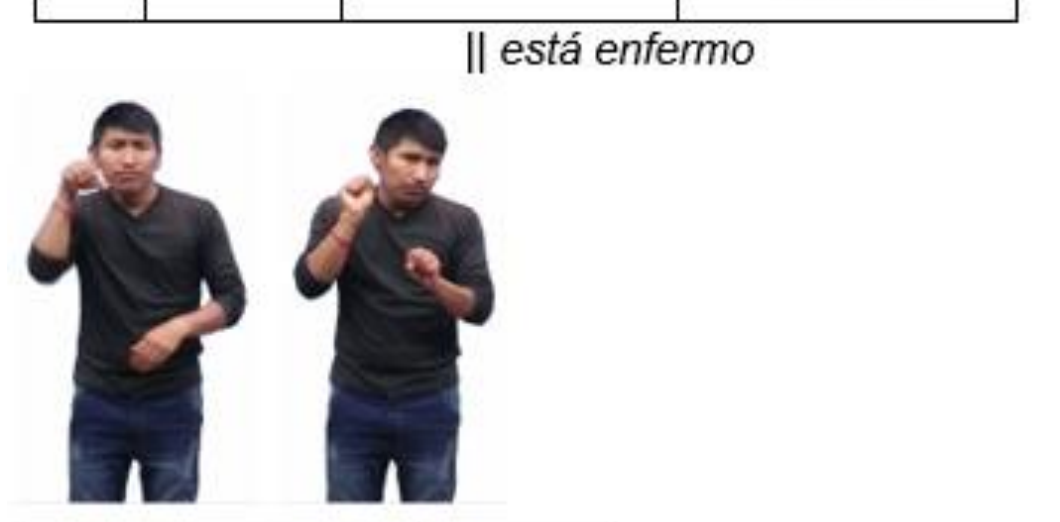

\begin{tabular}{|c|c|}
\hline $\begin{array}{l}\text { Cabezalnclzq } 2 \\
\rightarrow \text { Cabezalnclzq } 3 \\
\text { CeñoFruncido } 1 \\
\text { OjoAbierto } \\
\text { C+ } \\
\text { LabioApretado }\end{array}$ & [Reposo] \\
\hline POSIBLE & \\
\hline POSIBLE & \\
\hline $\begin{array}{c}\text { Adjunto } \\
\text { Modal } \\
\text { (Probabilidad) }\end{array}$ & \\
\hline
\end{tabular}

Figura 10. Toma 11B: Hipótesis marcada con recurso léxico equivalente a "posiblemente" como Adjunto Modal, con rasgos no manuales de Cabeza inclinada, Ojos abiertos y Labios apretados (Informante B) 


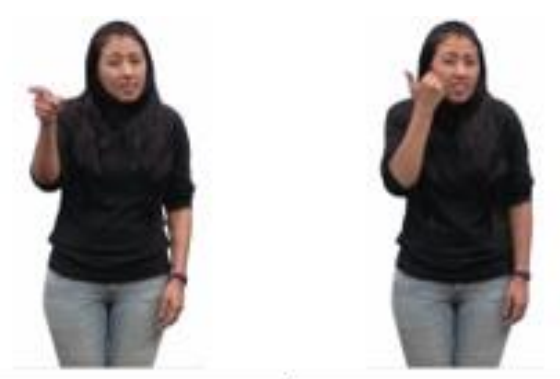

\begin{tabular}{|c|c|c|c|}
\hline RNM & \multirow{5}{*}{ [Reposo] } & $\begin{array}{l}\text { CuerpoinclAdelante } 1 \\
\text { MiradaDerecha } \\
\rightarrow \mathrm{C}+\end{array}$ & $\begin{array}{l}\text { CejaArqueada } 5 \\
\text { OjoSemicerrado } 3 \\
\text { C+ } \\
\text { g[e:] } \\
\text { [i.e. 'enfermo'] }\end{array}$ \\
\hline $\mathrm{MA}:$ & & $\begin{array}{l}\text { PRO-3sg } \\
\text { [pV2cara] }\end{array}$ & ENFERMO \\
\hline \multirow[t]{3}{*}{ MP: } & & & \\
\hline & & \multirow[t]{2}{*}{$\begin{array}{l}\text { Portador: } \\
\text { Poseedor }\end{array}$} & $\begin{array}{c}\text { Proceso: Rel. } \\
\text { Intensivo } \\
\text { (Atribución) }\end{array}$ \\
\hline & & & Atributo \\
\hline
\end{tabular}

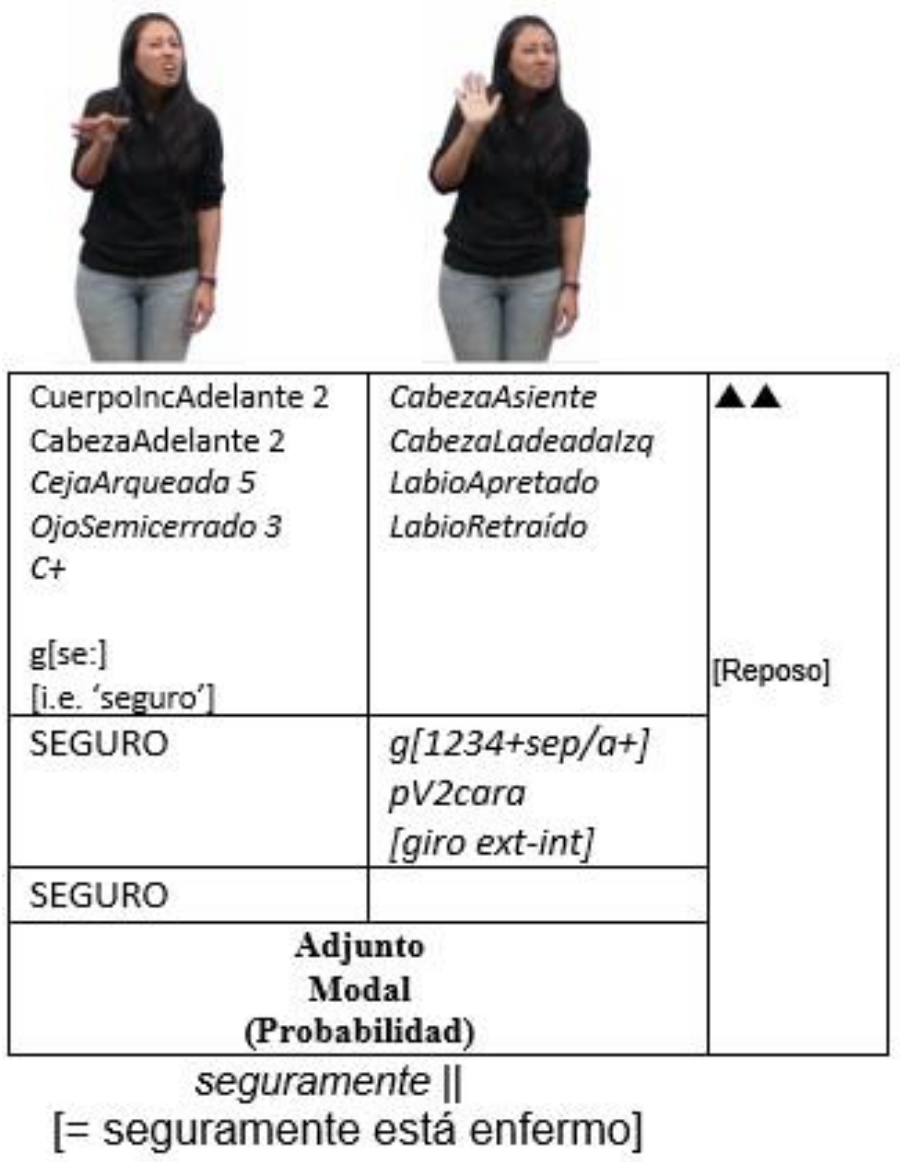

Figura 11. Toma 9A: Hipótesis marcada durante todo el enunciado mediante rasgos no manuales varios y Adjunto Modal compuesto por recurso léxico equivalente a "seguramente" acompañado de gesto unimanual (Informante C) 


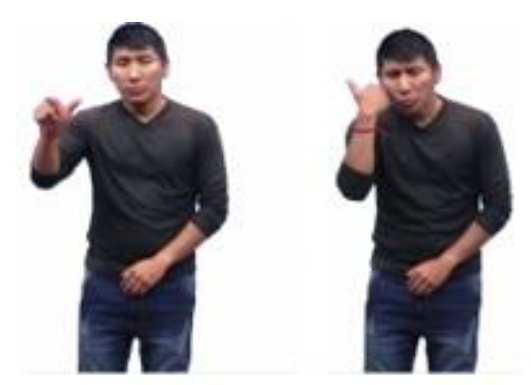

\begin{tabular}{|c|c|c|c|}
\hline RNM & \multirow{5}{*}{ [Reposo] } & $\begin{array}{l}\text { g[u:] } \\
\text { [i.e. 'tú', marca } \\
\text { idiosincrásica de } \\
\text { pronombre] }\end{array}$ & $\begin{array}{l}\text { CabezalnclDerecha } \\
\text { CabezaLadeadaDer } 2 \\
\text { OjoSemicerrado } 4 \\
\text { [i.e. 'enfermo'] }\end{array}$ \\
\hline IMA: & & PRO-3sg & ENFERMO \\
\hline \multirow[t]{3}{*}{ MP: } & & & \\
\hline & & \multirow[t]{2}{*}{$\begin{array}{l}\text { Portador: } \\
\text { Poseedor }\end{array}$} & $\begin{array}{l}\text { Proceso: Rel. } \\
\text { Intensivo } \\
\text { (Atribución) }\end{array}$ \\
\hline & & & Atributo \\
\hline
\end{tabular}

||| está enfermo ||
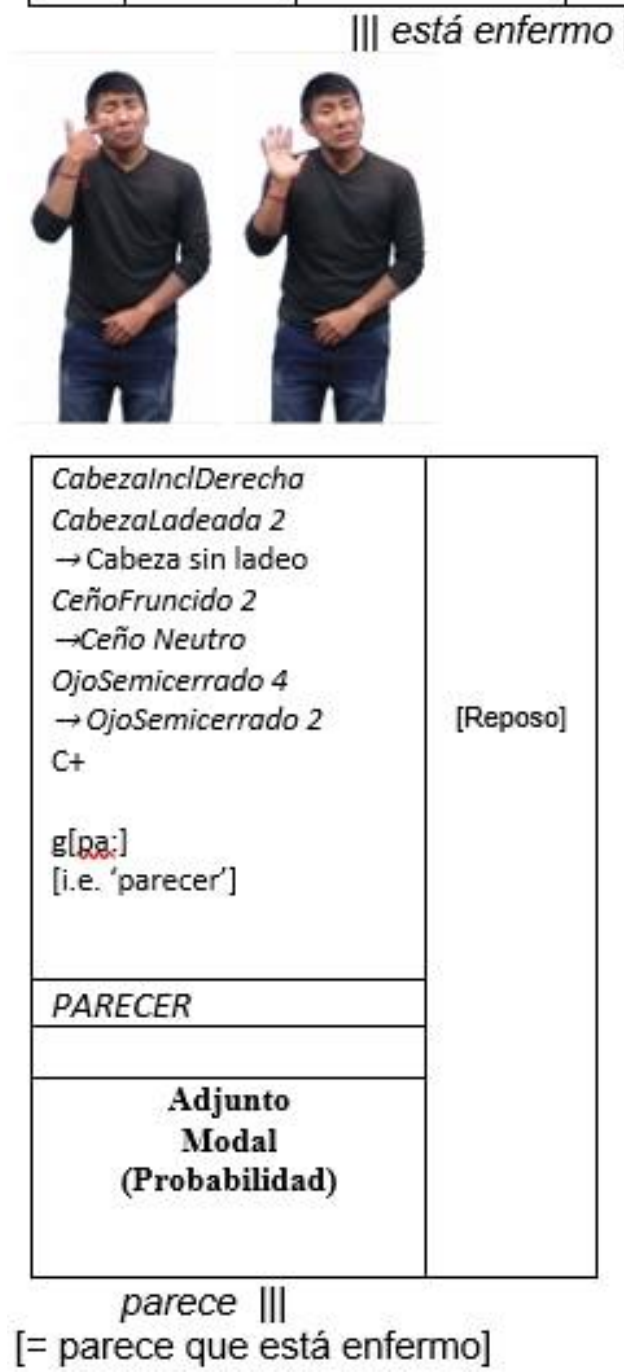

Figura 12. Toma 12A: Hipótesis marcada con Proceso Mental PARECER, acompañado de rasgos no manuales de Cabeza inclinada y ladeada, Ceño fruncido y

Ojos Semicerrados, que cambian (Informante B) 


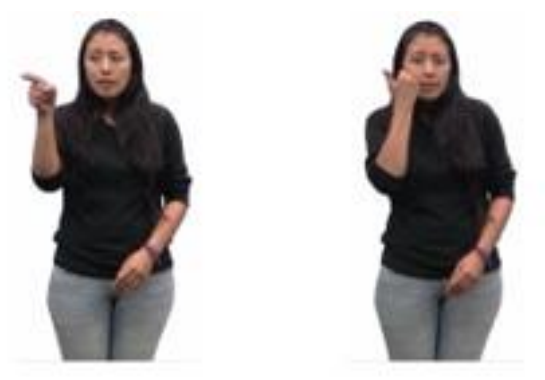

\begin{tabular}{|c|c|c|c|}
\hline RNM & \multirow{5}{*}{ [Reposo] } & $\begin{array}{l}\text { MiradaDerecha } \\
\text { [e:] } \\
\text { [i.e. 'él'?] }\end{array}$ & $\begin{array}{l}\text { C+ } \\
\text { OjoSemicerrado } 3 \\
\text { [i.e. 'enfermo'] }\end{array}$ \\
\hline MA: & & $\begin{array}{l}\text { PRO-3sg } \\
\text { [pV2cara] }\end{array}$ & ENFERMO \\
\hline \multirow[t]{3}{*}{ MP: } & & & \\
\hline & & $\begin{array}{l}\text { Portador: } \\
\text { Poseedor }\end{array}$ & $\begin{array}{c}\text { Proceso: Rel. } \\
\text { Intensivo } \\
\text { (Atribución) }\end{array}$ \\
\hline & & & Atributo \\
\hline
\end{tabular}

II él está enfermo

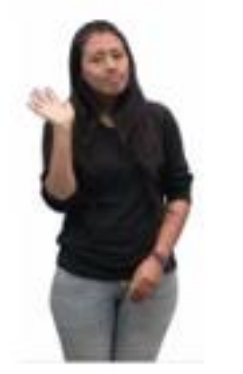

\begin{tabular}{|c|c|}
\hline $\begin{array}{l}\text { CabezaLadDer } \times 2 \\
\text { CabezaLadlzq } \\
\text { CabezalnclDer } \\
\rightarrow \text { Cabladlzq } \\
\rightarrow \text { Neutra } \\
\text { LabioApretado } \\
\text { [i.e. 'no es seguro'] }\end{array}$ & [Reposo] \\
\hline $\begin{array}{l}g[1234+\operatorname{sep} / a+] \\
{[\mathrm{pV} 2 \text { cara] }}\end{array}$ & \\
\hline $\begin{array}{c}\text { Adjunto } \\
\text { Modal } \\
\text { (Probabilidad) }\end{array}$ & \\
\hline
\end{tabular}

Figura 13. Toma 7: Hipótesis marcada por gesto unimanual como Adjunto Modal, acompañado de rasgos no manuales de Cabeza ladeada e inclinada y Labios apretados (Informante C) 

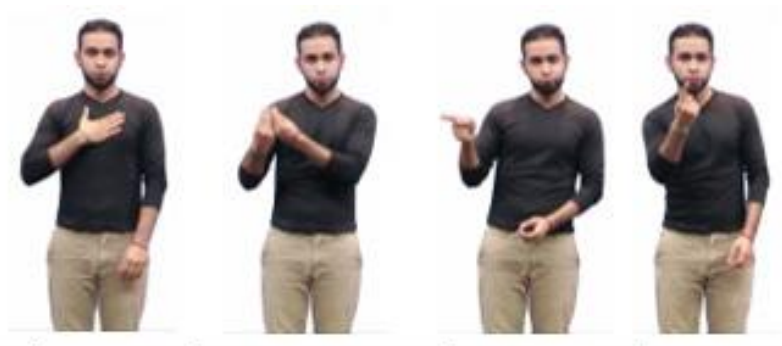

\begin{tabular}{|c|c|c|c|c|c|}
\hline RNM & \multirow[b]{4}{*}{ [Reposo] } & $\mathrm{C}+$ & $\begin{array}{l}\text { CabezalnclDer } \\
\mathrm{C}+\end{array}$ & $\mathrm{C}+$ & $\begin{array}{l}\text { CuerpolnclDer } \\
\mathrm{C}+\end{array}$ \\
\hline MA: & & Pos-1sg & COMPAÑERO & PRO-3sg & DECIR \\
\hline \multirow[t]{2}{*}{ MP: } & & & COMPAÑERO & & \\
\hline & & \multicolumn{3}{|c|}{ Locutor } & $\begin{array}{c}\text { Proceso: } \\
\text { Verbal }\end{array}$ \\
\hline
\end{tabular}
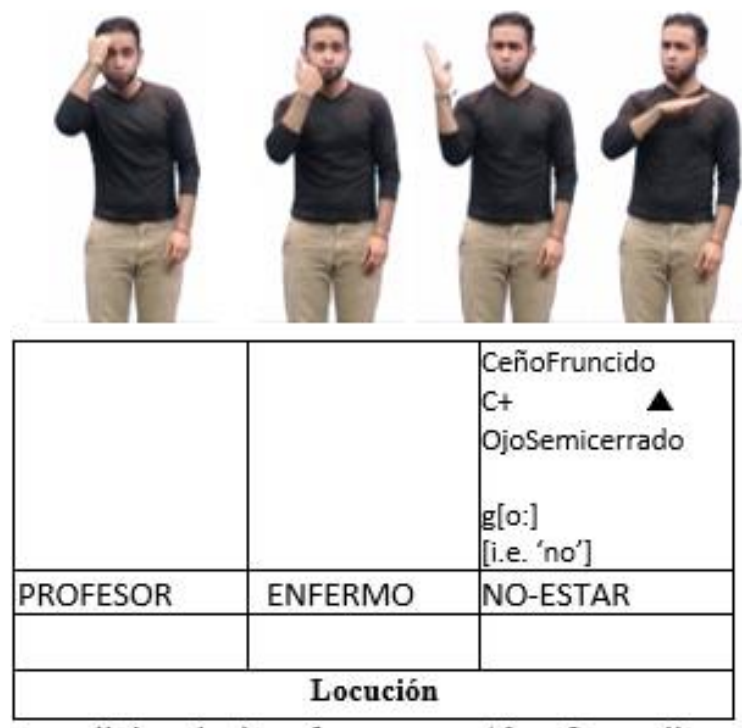

|| (que) el profesor no está enfermo \|

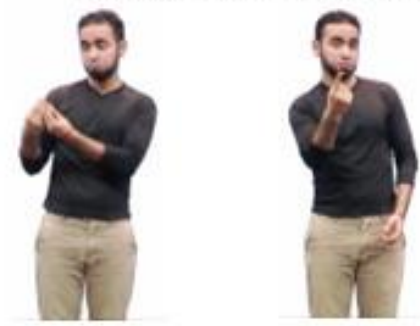

\begin{tabular}{|l|l|l|}
\hline $\begin{array}{l}\text { CejaArqueado } \\
\text { MiradaAbajoDerecha } \\
\text { [i.e. al compañero] } \\
\text { LabioRetraído }\end{array}$ & C+ & \\
\hline COMPAÑERO & DECIR & \\
\hline [Reposo]
\end{tabular}

$$
\text { él (lo) dice III }
$$

[=mi compañero dice que el profesor no está enfermo]

Figura 14. Toma 18: Hipótesis marcada mediante Evidencial con DECIR, que implica incertidumbre sobre la veracidad de la proposición (Informante A) 


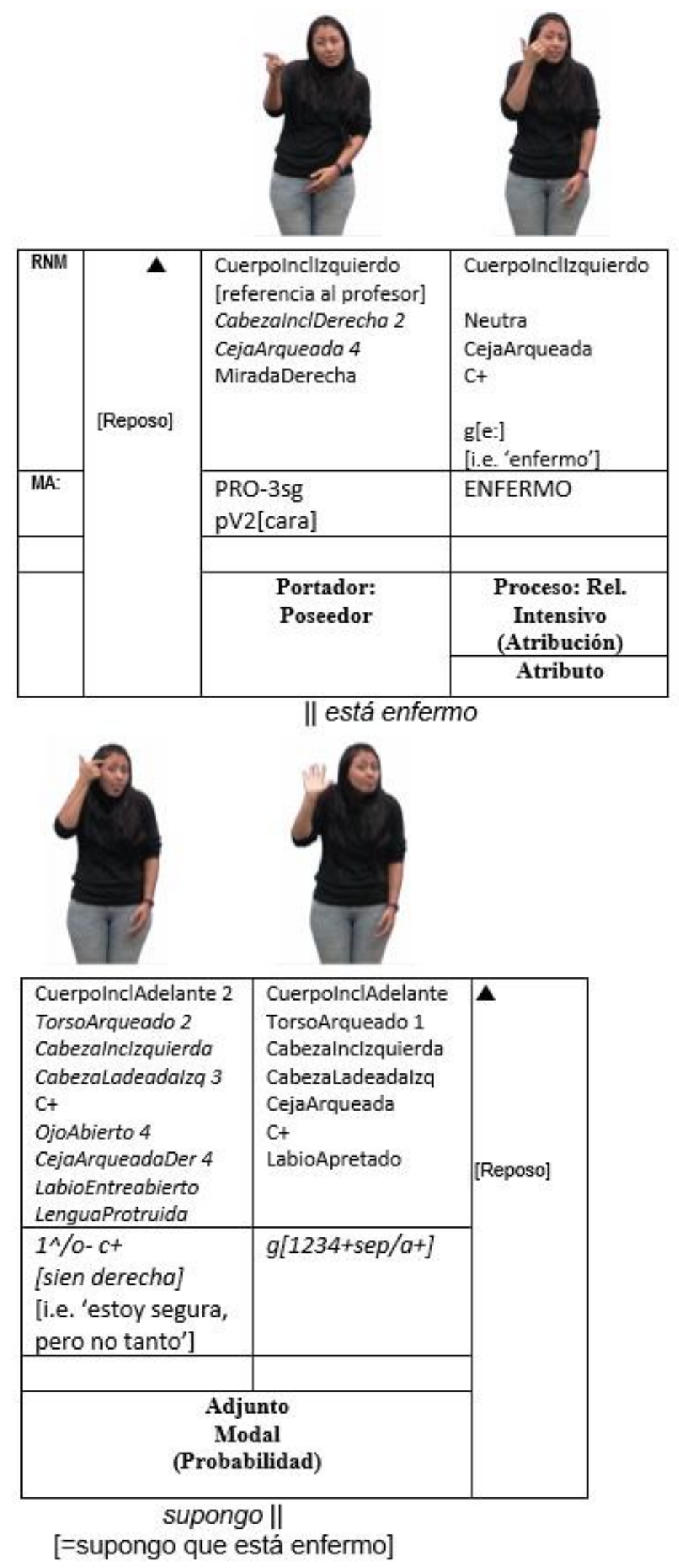

Figura 15. Toma 9C: Hipótesis marcada mediante Adjunto Modal conformado por gesto unimanual acompañado de rasgos no manuales de Cuerpo inclinado, Torso arqueado, Cabeza ladeada e inclinada, Cejas arqueadas, Ojos abiertos y Lengua protruida, anticipado por rasgos no manuales de Cabeza inclinada y Cejas arqueadas desde el comienzo del enunciado (Informante C) 


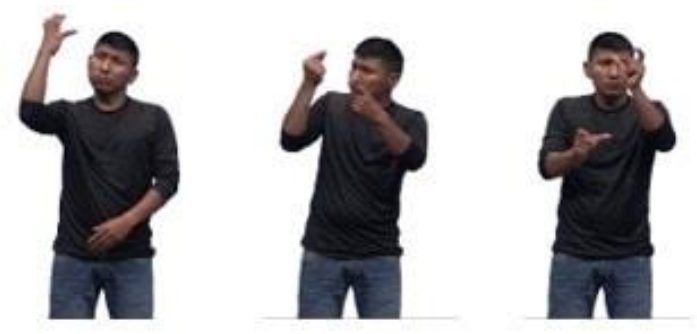

\begin{tabular}{|c|c|c|c|c|}
\hline RNM & \multirow{4}{*}{ [Reposo] } & $\begin{array}{l}\text { CeñoFruncido } 5 \\
\text { LabioSopla }\end{array}$ & & CeñoFruncido 4 \\
\hline MA: & & $\begin{array}{l}\text { HACE-MUCHO- } \\
\text { TIEMPO }\end{array}$ & SIGLO & PERSONA[pl] \\
\hline \multirow[t]{2}{*}{ MP: } & & $\begin{array}{l}\text { HACE-MUCHO- } \\
\text { TIEMPO }\end{array}$ & SIGLO & PERSONA[pl] \\
\hline & & \multicolumn{2}{|c|}{$\begin{array}{c}\text { Circunstancia: } \\
\text { Localización } \\
\text { (temporal) }\end{array}$} & Perceptor \\
\hline
\end{tabular}

III durante siglos muchas personas

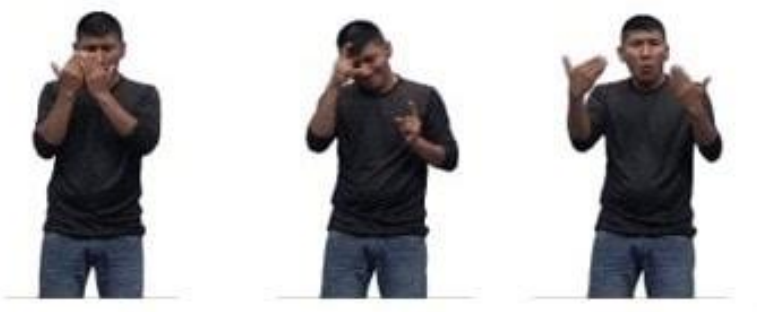

\begin{tabular}{|l|l|l|}
\hline CabezaAdelante & CeñoFruncido 5 & CeñoFruncido 2 \\
\hline $\begin{array}{l}\text { ESTUDIAR- } \\
\text { RIGUROSAMENTE }\end{array}$ & $\begin{array}{l}\text { RAZONAR[li.e. } \\
\text { PREGUNTARSE] }\end{array}$ & COMO \\
\hline $\begin{array}{l}\text { ESTUDIAR- } \\
\text { RIGUROSAMENTE }\end{array}$ & $\begin{array}{l}\text { RAZONAR[li.e } \\
\text { PREGUNTARSE] }\end{array}$ & \\
\hline \multicolumn{1}{|c|}{$\begin{array}{c}\text { Proceso: } \\
\text { Mental }\end{array}$} & $\begin{array}{c}\text { Proceso: } \\
\text { Mental/Verbal }\end{array}$ & Fenómeno \\
\hline
\end{tabular}

han estudiado y se han preguntado \| cómo
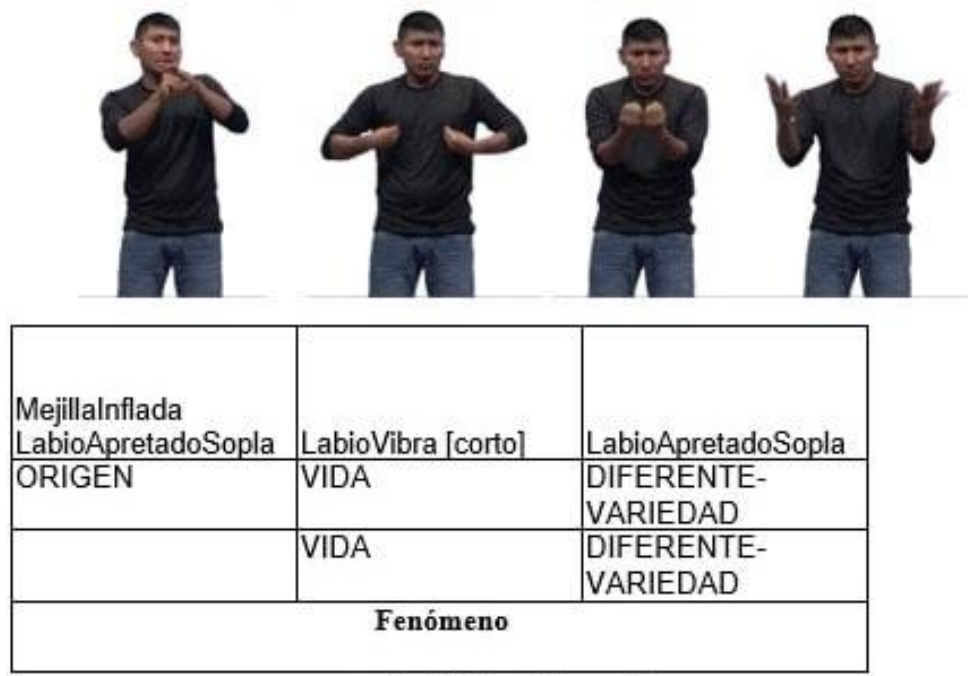

se originó la vida, ... III

Figura 16. Toma 69: Hipótesis marcada mediante Adjunto Modal con Proceso Mental/Verbal RAZONAR, acompañado de rasgo no manual de Ceño fruncido (Informante B) 


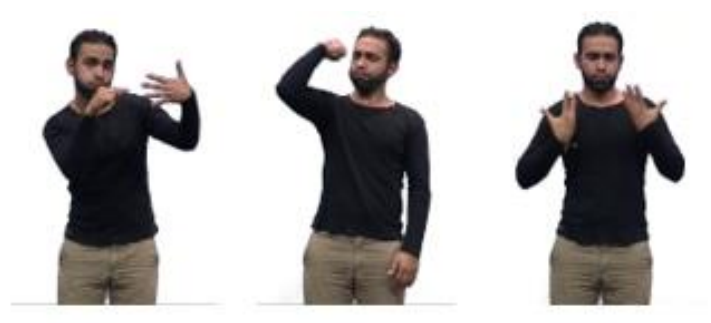

\begin{tabular}{|c|c|c|c|c|}
\hline RNM & \multirow{4}{*}{ [Reposo] } & & $\begin{array}{l}\text { CeñoFruncido } 5 \\
\text { LabioSopla }\end{array}$ & \\
\hline MA: & & $\begin{array}{l}\text { LISTA-DE- } \\
\text { MÁS-DE- } \\
\text { CINCO } \\
1+/ 0-\text { señala } \\
\text { dígitos MP sin } \\
\text { contacto }\end{array}$ & $\begin{array}{l}\text { HACE-MUCHO- } \\
\text { TIEMPO }\end{array}$ & VIDA \\
\hline \multirow[t]{2}{*}{ MP: } & & $\begin{array}{l}\text { LISTA-DE- } \\
\text { MÁS-DE- } \\
\text { CINCO } \\
1234+\text { sep/a+ }\end{array}$ & $\begin{array}{l}\text { HACE-MUCHO- } \\
\text { TIEMPO }\end{array}$ & VIDA \\
\hline & & $\begin{array}{c}\text { Proceso: } \\
\text { Existencial } \\
\text { [Fxistentel }\end{array}$ & $\begin{array}{l}\text { Circunstancia: } \\
\text { Localización } \\
\text { (temporal) }\end{array}$ & Actor \\
\hline
\end{tabular}

III hay varias [hipótesis] || hace mucho tiempo la vida

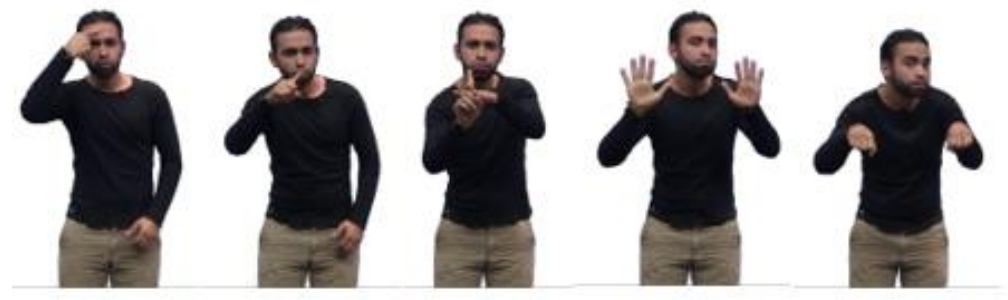

\begin{tabular}{|c|c|c|c|c|}
\hline & $\begin{array}{l}\text { LabioApretado } \\
\text { LabioSopla }\end{array}$ & CabezaLadlzq & $\begin{array}{l}\text { CabezaLadlizq } \\
\text { CejaArqueada } 4 \\
\text { LabioRetraído }\end{array}$ & $\boldsymbol{\Delta}$ \\
\hline \multirow[t]{2}{*}{ SOLO } & ORIGINARSE & $g[1234+\operatorname{sep} / a+]$ & PODER & \multirow{4}{*}{ [Reposo] } \\
\hline & ORIGINARSE & $g[1234+\operatorname{sep} / a+]$ & PODER & \\
\hline \multirow[t]{2}{*}{$\begin{array}{c}\text { Circunstancia: } \\
\text { Manera } \\
\text { (Cualidad) }\end{array}$} & Predicador & $\begin{array}{c}\text { Adjunto: } \\
\text { Modal } \\
\text { (probabilidad) }\end{array}$ & $\begin{array}{c}\text { Finito: } \\
\text { Modalidad } \\
\text { (Modalización: } \\
\text { Probabilidad) } \\
\end{array}$ & \\
\hline & \multicolumn{3}{|c|}{$\begin{array}{l}\text { Proceso: } \\
\text { Material }\end{array}$} & \\
\hline
\end{tabular}

pudo haberse originado por sí sola III

Figura 17. Toma 71: Hipótesis marcada mediante gesto bimanual 1234+sep/a+ con sentido del Finito modal "poder" (Informante A)

\section{Nivel Bajo de probabilidad}

Por último, el nivel Bajo viene marcado léxicamente con Procesos Mentales como CREER o NO-SABER, con Evidenciales introducidos por DECIR o con el gesto unimanual o bimanual (1234+sep/a+), pero con combinaciones de rasgos no manuales de baja probabilidad, algunos de los cuales, de hecho salen solo en este nivel (CabezaAsiente, HombroArriba, OjoAbierto, LabioRetraído y MandíbulaDerecha, como se ve en las Figuras 18, 19, 20 y 21: 


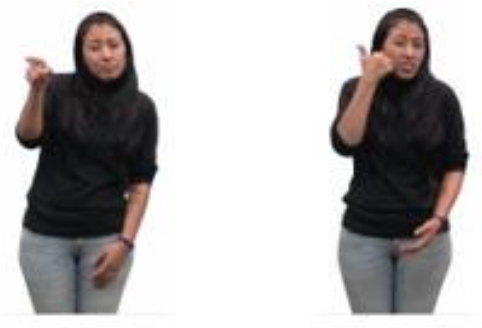

\begin{tabular}{|c|c|c|c|}
\hline RNM & \multirow{5}{*}{ [Reposo] } & $\begin{array}{l}\text { CuerpolnclinadaDer1 } \\
\text { CejaArqueada } \\
\text { MiradaDerecha }\end{array}$ & $\begin{array}{l}\text { Cuerpolnclinadolzq } 2 \\
\text { Cabezalnclinadalzq2 } \\
\text { C+ } \\
\text { [e:] } \\
\text { [i.e. 'enfermo'] }\end{array}$ \\
\hline MA: & & $\begin{array}{l}\text { PRO-3sg } \\
\text { [pV2cara] }\end{array}$ & ENFERMO \\
\hline \multirow[t]{3}{*}{ MP: } & & & \\
\hline & & \multirow[t]{2}{*}{$\begin{array}{l}\text { Portador: } \\
\text { Poseedor }\end{array}$} & $\begin{array}{c}\text { Proceso: Rel. } \\
\text { Intensivo } \\
\text { (Atribución) }\end{array}$ \\
\hline & & & Atributo \\
\hline
\end{tabular}

|ll está enfermo ||

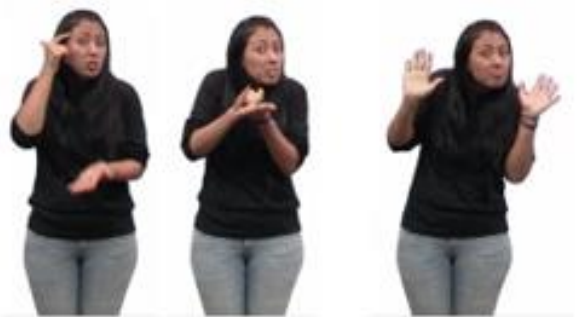

\begin{tabular}{|c|c|c|}
\hline $\begin{array}{l}\text { Cuerpolnclinadolzq } 2 \\
\text { HombroArriba } 3 \\
\text { CabezaLadeadalzq } 2 \\
\text { CejaArqueada } 5 \\
\text { C+ } \\
\rightarrow \text { OjoAbierto } 5 \\
\text { g[o:] } \\
\text { [i.e. 'yo'] }\end{array}$ & $\begin{array}{l}\text { Cuerpoinclinadolzq } 4 \\
\text { CabezaLadeadalzq } 4 \\
\text { C+ } \\
\text { LabioApretado }\end{array}$ & 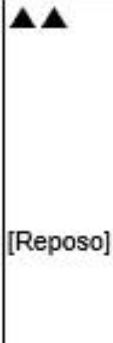 \\
\hline CREER & $g[1234+\operatorname{sep} / a+]$ & \\
\hline CREER & $g[1234+\operatorname{sep} / a+]$ & \\
\hline \multicolumn{2}{|c|}{$\begin{array}{c}\text { Adjunto } \\
\text { Modal } \\
\text { (Probabilidad) }\end{array}$} & \\
\hline
\end{tabular}

yo creería |||

[= yo creería que está enfermo]

Figura 18. Toma 9B: Hipótesis marcada mediante Adjunto Modal con Proceso Mental CREER, acompañado de rasgos no manuales de Cuerpo inclinado, Hombros arriba, Cabeza ladeada, Ceja arqueada y Ojos que se abren, todo seguido de gesto bimanual 1234+sep/a+, que disminuye la probabilidad (Informante C) 


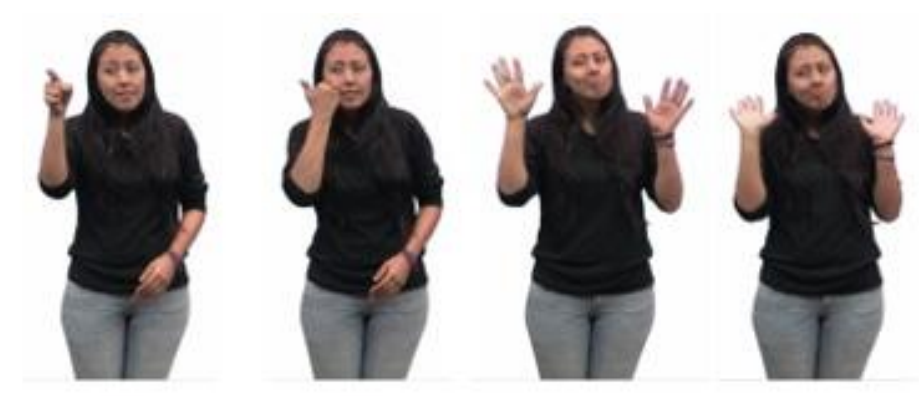

\begin{tabular}{|c|c|c|c|c|c|}
\hline RNM & \multirow{5}{*}{ [Reposo] } & & $\begin{array}{l}\text { MiradaDerecha } \\
\text { [i.e. al profesor] }\end{array}$ & $\begin{array}{l}\text { CabezaLadeadaDer } \\
\text { CabezaAsiente } \\
\text { CeñoFruncido } 2 \\
\text { LabioApretado } \\
\text { MandibulaDer }\end{array}$ & \multirow{5}{*}{ [Reposo] } \\
\hline MA: & & $\begin{array}{l}\text { PRO-3sg } \\
\text { [pV2cara] }\end{array}$ & ENFERMO & $\begin{array}{l}g[1234+\operatorname{sep} / a+] \\
\text { pV2cara } \\
\text { Rot. int-ext }\end{array}$ & \\
\hline \multirow[t]{3}{*}{ MP: } & & & & $\begin{array}{l}g[1234+\operatorname{sep} / a+] \\
\text { pV2cara } \\
\text { [contralateral] }\end{array}$ & \\
\hline & & \multirow[t]{2}{*}{$\begin{array}{l}\text { Portador: } \\
\text { Poseedor }\end{array}$} & $\begin{array}{c}\text { Proceso: Rel. } \\
\text { Intensivo } \\
\text { (Atribución) }\end{array}$ & \multirow[t]{2}{*}{$\begin{array}{c}\text { Adjunto } \\
\text { Modal } \\
\text { (Probabilidad) }\end{array}$} & \\
\hline & & & Atributo & & \\
\hline
\end{tabular}

\| él podría estar enfermo \|

Figura 19. Toma 6: Hipótesis marcada mediante Adjunto Modal constituido por gesto bimanual 1234+sep/a+, acompañado de rasgos no manuales de Labios apretados y Mandíbula desplazada hacia un lado, lo que disminuye la probabilidad (Informante C) 


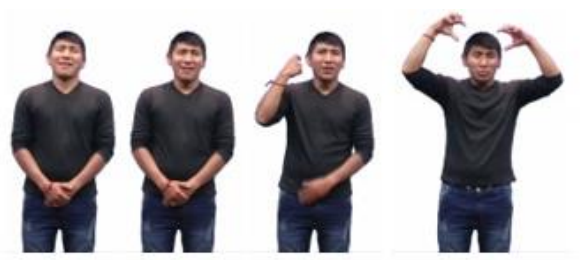

\begin{tabular}{|c|c|c|c|c|}
\hline RNM & \multirow[t]{5}{*}{ [Reposo] } & $\begin{array}{l}\text { CabezaAsiente } \\
\text { MiradaArriba } \\
\text { CeñoFruncido } \\
\text { g[a:] } \\
\text { [i.e. 'iah!'] }\end{array}$ & & $\begin{array}{l}\text { Ceñofruncido } 2 \\
\text { LabioSopla }\end{array}$ \\
\hline MA: & & & AYER & LLOVER \\
\hline \multirow[t]{3}{*}{ MP: } & & & & \\
\hline & & $\begin{array}{c}\text { Adjunto: } \\
\text { Modal }\end{array}$ & $\begin{array}{c}\text { Circunstancia } \\
:\end{array}$ & \multirow[t]{2}{*}{$\begin{array}{l}\text { Proceso: } \\
\text { Material }\end{array}$} \\
\hline & & (Comentario) & $\begin{array}{c}\begin{array}{c}\text { Localización } \\
\text { (temporal) }\end{array} \\
\end{array}$ & \\
\hline
\end{tabular}

|l| ah || ayer llovió ||

\begin{tabular}{|c|c|}
\hline $\begin{array}{l}\text { CeñoFruncido } 2 \\
\text { g[o:] } \\
\text { [i.e. 'todo'] }\end{array}$ & CeñoFruncido 2 \\
\hline $\begin{array}{l}\text { CL:1234+sep/a+ } \\
\text { [agua corriendo } \\
\text { por el cuerpo del } \\
\text { profesor] }\end{array}$ & MOJADO \\
\hline $\begin{array}{l}\text { CL:1234+sep/a+ } \\
\text { [agua corriendo } \\
\text { por el cuerpo del } \\
\text { profesor] }\end{array}$ & MOJADO \\
\hline $\begin{array}{l}\text { Proceso: } \\
\text { Material }\end{array}$ & $\begin{array}{c}\text { Proceso: Rel. } \\
\text { Intensivo } \\
\text { (Atribución) }\end{array}$ \\
\hline $\begin{array}{c}\text { Circunstancia: } \\
\text { Localización } \\
\text { (espacial) }\end{array}$ & Portador \\
\hline
\end{tabular}

|| se mojó todo \|

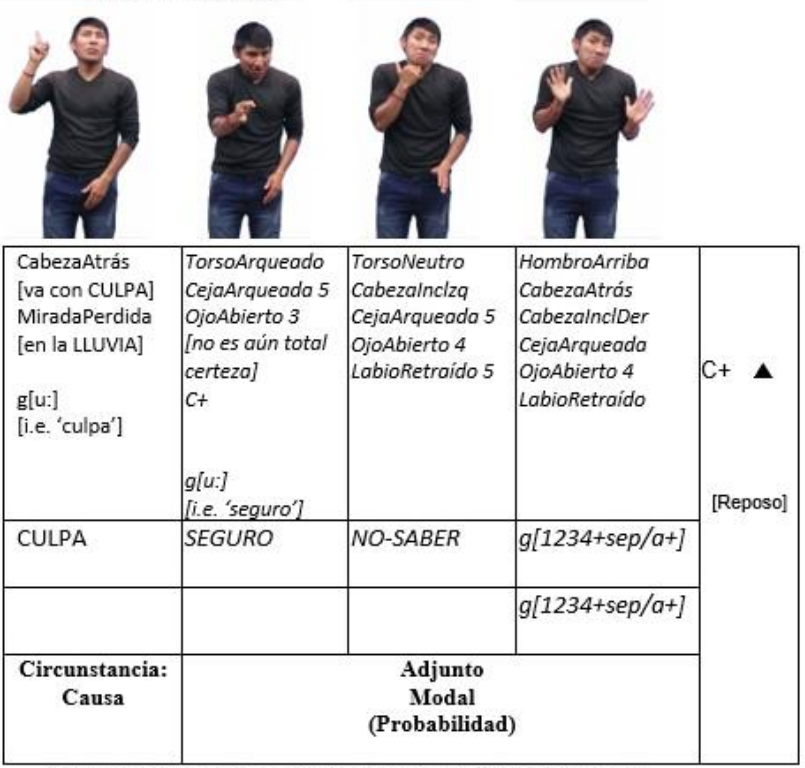

|| debe haber sido por eso || pero no estoy seguro |||

Figura 20. Toma 17: Hipótesis marcada mediante Adjunto Modal constituido por recursos léxicos y sus respectivos rasgos no manuales, más gestos bimanuales

(Informante B) 


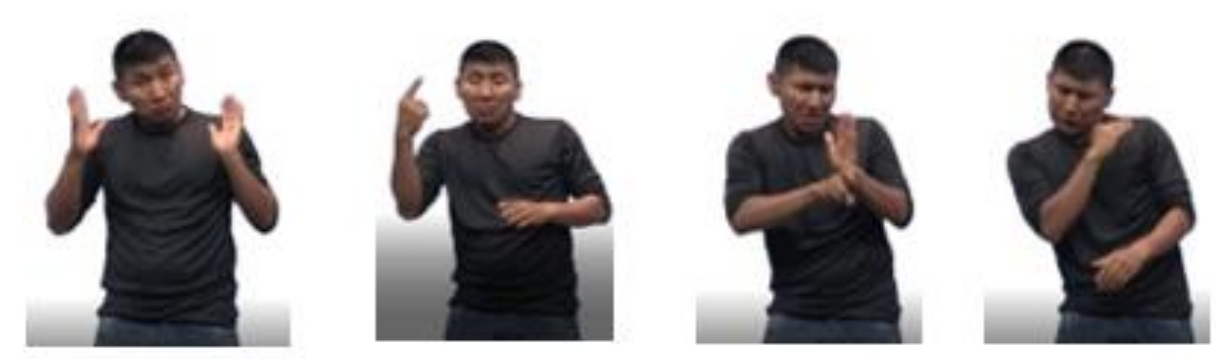

\begin{tabular}{|l|l|l|l|}
\hline $\begin{array}{l}\text { CabezalncDer } \\
\text { CabezaLadlzq } \\
\text { CejaArqueada 4 } \\
\text { BarbillaAdelante 3 }\end{array}$ & $\begin{array}{l}\text { CejaArqueada 4 } \\
\text { MiradaArribaDer } \\
\text { [i.e. referencia a la } \\
\text { hipótesis] } \\
\text { LabioRetraido 3 } \\
g[e]\end{array}$ & $\begin{array}{l}\text { CejaArqueada 4 } \\
\text { LabioRetraído 3 }\end{array}$ & $\begin{array}{l}\text { CuerpolncDer } \\
\text { CabezaMueve }\end{array}$ \\
\hline $\mathrm{g}[1234+/ \mathrm{a}+]$ & $\begin{array}{l}\text { DECIR } \\
\text { [pV2cara] }\end{array}$ & $\begin{array}{l}\text { TEORIA } \\
\times 2\end{array}$ & NO-SER \\
\hline $\mathrm{g}[1234+/ \mathrm{a}+]$ & & $\begin{array}{l}\text { TEORIA } \\
\times 2\end{array}$ & NO-SER \\
\hline \multicolumn{1}{|c|}{ Adjunto } & \multicolumn{1}{|c|}{$\begin{array}{l}\text { Portador: } \\
\text { Atributo }\end{array}$} & $\begin{array}{c}\text { Proceso: } \\
\text { Relacional } \\
\text { Conjuntivo } \\
\text { (atribución) }\end{array}$ \\
\hline
\end{tabular}

Figura 21. Toma 73: Hipótesis marcada mediante gesto bimanual 1234+/a+ y recurso léxico POSIBLE (Informante B)

Las cláusulas condicionales como marcación de hipótesis

Asimismo, al igual que en las lenguas orales, como lo postula Lock (1996), las cláusulas condicionales pueden ser también en la LSC un recurso para indicar hipótesis, como se ve en las Figuras 22 y 23: 

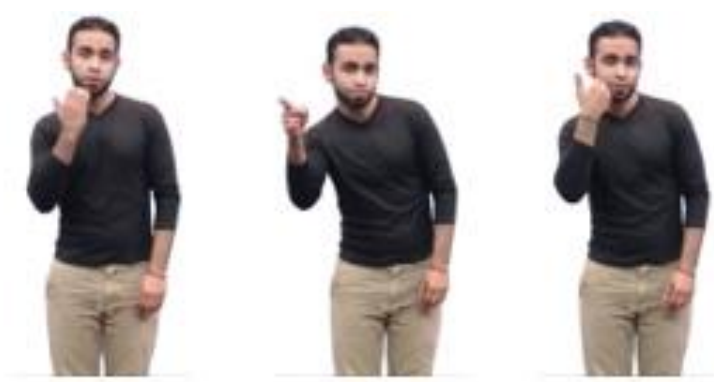

\begin{tabular}{|c|c|c|c|c|}
\hline RNM & \multirow{4}{*}{ [Reposo] } & $\mathrm{C}+$ & $\begin{array}{l}\text { CuerpolnclDer } \\
\text { CuerpolnclAde } \\
\text { C+ }\end{array}$ & $\mathrm{C}+$ \\
\hline MA: & & EJEMPLO & PRO-3sg & ENFERMO \\
\hline \multirow[t]{2}{*}{ MP: } & & & & \\
\hline & & \multicolumn{3}{|c|}{$\begin{array}{c}\text { Adjunto } \\
\text { Modal } \\
\text { (Probabilidad) }\end{array}$} \\
\hline
\end{tabular}

||| si él está enfermo, ||
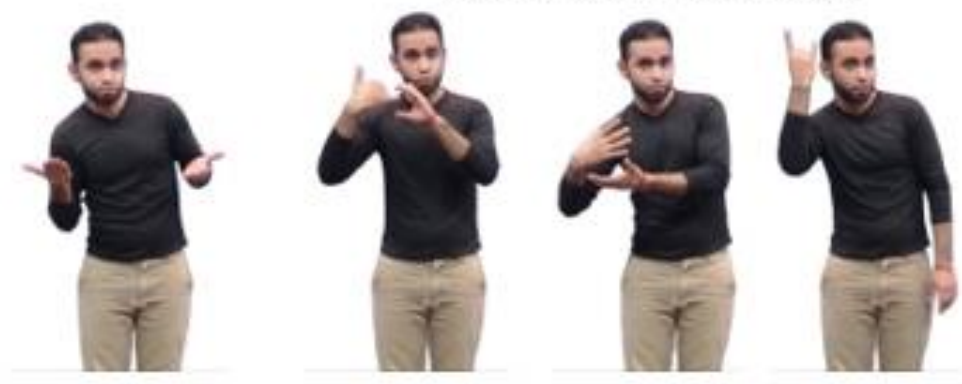

\begin{tabular}{|c|c|c|c|c|}
\hline $\begin{array}{l}\text { CuerpoinclDer } \\
\text { CuerpoinclAde } \\
\text { C+ } \\
\text { LabioApretado }\end{array}$ & $\mathrm{C}+$ & $\begin{array}{l}\text { Cuerpoinc/Der } \\
\text { CuerpoinclAde }\end{array}$ & & \multirow{5}{*}{ [Reposo] } \\
\hline$g[1234+\operatorname{sep} / a+]$ & WHATSAPP & $\begin{array}{l}\text { ENVIAR- } \\
\text { MENSAJE }\end{array}$ & ALGO & \\
\hline$g[1234+\operatorname{sep} / a+]$ & WHATSAPP & $\begin{array}{l}\text { ENVIAR- } \\
\text { MENSAJE }\end{array}$ & & \\
\hline \multirow{2}{*}{$\begin{array}{c}\text { Adjunto } \\
\text { Modal } \\
\text { (Probabilidad) }\end{array}$} & \multirow{2}{*}{$\begin{array}{c}\text { Circunstancia: } \\
\text { Modo } \\
\text { (medio) }\end{array}$} & Actor & \multirow[t]{2}{*}{ Objeto } & \\
\hline & & $\begin{array}{l}\text { Proceso: } \\
\text { Material }\end{array}$ & & \\
\hline
\end{tabular}

|| entonces envía algún mensaje por WhatsApp |||

Figura 22. Toma 22: Hipótesis marcada por cláusula condicional real de predicción, introducida por la seña EJEMPLO (Informante A) 


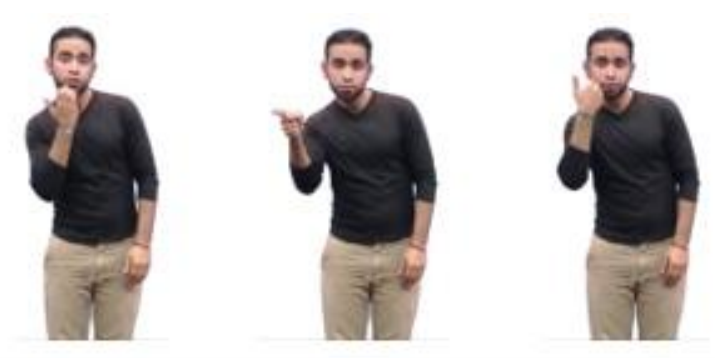

\begin{tabular}{|c|c|c|c|c|}
\hline RNM & \multirow{4}{*}{ [Reposo] } & $\begin{array}{l}\text { CuerpolncDer } \\
\text { CuerpolncAde }\end{array}$ & $\begin{array}{l}\text { Neutro } \\
\rightarrow \text { CuerpolncDer } \\
\text { CuerpolncAde }\end{array}$ & $\begin{array}{l}\text { CuerpolncDer } \\
\text { CuerpolncAde }\end{array}$ \\
\hline MA: & & EJEMPLO & PRO-3sg & ENFERMO \\
\hline \multirow[t]{2}{*}{ MP: } & & & & \\
\hline & & \multicolumn{3}{|c|}{$\begin{array}{c}\text { Adjunto } \\
\text { Modal } \\
\text { (Probabilidad) }\end{array}$} \\
\hline
\end{tabular}

||| si él estuviera enfermo \|

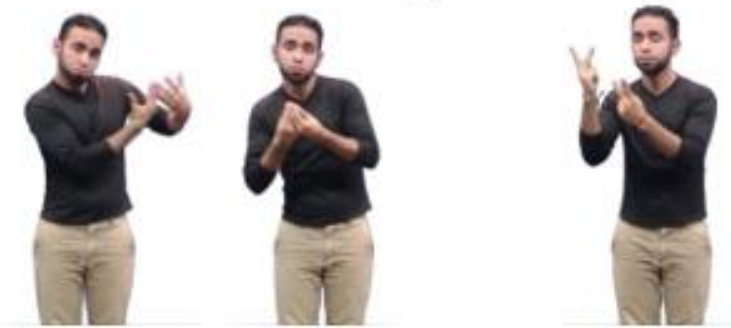

\begin{tabular}{|c|c|c|}
\hline & $\begin{array}{l}\text { CuerpoLadDer } \\
\text { CabezaLadDer } \\
\text { MiradaDer }\end{array}$ & \\
\hline $\begin{array}{l}\text { CL:1234crz } \alpha \text { SE-MUEVE- } \\
\text { DE[pV3pecho contralatera]_-A[pVOpecho] }\end{array}$ & VISITAR & \\
\hline $\begin{array}{l}\text { CL: } 1234 c r z \alpha \text { SE-MUEVE- } \\
\text { DE[p/3pecho contralatera] } \text { A }^{\text {[pVopecho] }}\end{array}$ & VISITAR & [Reposo] \\
\hline Actor & \multirow{3}{*}{$\begin{array}{l}\text { Proceso } \\
\text { Material }\end{array}$} & \\
\hline Objeto & & \\
\hline $\begin{array}{l}\text { Proceso: } \\
\text { Material }\end{array}$ & & \\
\hline
\end{tabular}

|| habría que ir a visitarlo |||

Figura 23. Toma 28: Hipótesis marcada mediante cláusula condicional irreal de deducción, introducida por la seña EJEMPLO (Informante A)

El Informante A prefirió construir sus cláusulas condicionales con la expresión EJEMPLO, una de las posibilidades que existen también en español oral ("un ejemplo, tú vas a la playa en tiempo de brisa y te asustas con el oleaje" = "si vas a la playa en tiempo de brisa, te asustas con el oleaje"). Sin embargo, no es la única forma de expresar la condición en LSC. López y Osorno (2004), en efecto, dan ejemplos en que los rasgos no manuales juegan un papel muy importante. Estas autoras afirman, de hecho, que 
“teniendo en cuenta la descripción hecha por Valli y Lucas [1995], se ha observado un marcador no manual equivalente al IF del inglés" (López y Osorno, 2004, p. 187). Por lo que se puede ver en sus dos ejemplos, ya que el metalenguaje es ligeramente diferente, se trata de los rasgos no manuales de CuerpoInclinadoAtrás, CejaArqueada, OjoAbierto y LabioRetraído para las condicionales negativas (si no me pagan, no puedo ir al paseo), pero puede incluir CuerpoInclinadoAdelante con CabezaAsiente, además de los otros rasgos no manuales, en las condicionales afirmativas (si me pagan, te acompañaré). En esto puede verse similitud con la expresión de muchas condicionales en español oral, por lo menos en ejemplos surgidos de introspección. Pero hay que tener en cuenta también, como se ve en los ejemplos de las Figuras 22 y 23, que los rasgos no manuales de Cuerpo, Cabeza y Mirada se ven afectados también por la expresión de la referencia anafórica, en este caso para reforzar la localización espacial del participante al que el señante se refiere, "el profesor", señalado por el ÍNDEX y, en el segundo ejemplo, también por los Procesos Materiales direccionales referidos a la necesidad de visitarlo.

\section{CONCLUSIONES}

En la LSC se pueden identificar también los tres niveles de probabilidad, Alto, Medio y Bajo, propuestos por Halliday (2014). Tal como lo indican Lavid et al. (2010) para el español, posiblemente no todos los señantes de LSC estarán de acuerdo en la marcación de cada uno. Aquí llegamos a generalizaciones basadas en lo que señan y lo que indican nuestros informantes. Tanto en lenguas de señas como orales va a haber siempre idiosincrasia, por lo que una conclusión general no es siempre factible. Incluso, por tratarse de una lengua que se realiza en la modalidad viso-gestual, la LSC permitiría en la hipótesis, como lo hace en otros aspectos de la léxico-gramática, una expresión más fina de grados de probabilidad que los propuestos por Halliday (2014), Thompson (2014), Lavid et al. (2010) o Lock (1996) para lenguas orales como el inglés o el español. Esto es debido a la multiplicidad de articuladores, algunos manuales y otros faciales o corporales, que permiten combinaciones de señas léxicas y gramaticales, rasgos no manuales léxicos y gramaticales de la parte baja de la cara, así como rasgos no manuales de la parte superior de la cara. Estos últimos son posiblemente de origen gestual innato, pero parecen haberse ido gramaticalizando debido al canal de comunicación viso-gestual en que se realiza la lengua. Es posible, por supuesto, postular una descripción similar para el estudio de la hipótesis en lenguas orales, incluyendo la gestualidad facial y corporal, así como la prosodia y la proxemia, en cuyo caso el sistema descriptivo de tres o cuatro niveles, basado en la expresión oral organizada sintagmáticamente, resulta en demasía simplificado. Tal vez está todavía por hacerse una lingüística del "sonido-y-gesto" como la postulada por Hjelmslev (1943/1971).

Se vio, sin embargo, que se necesita en LSC la misma combinación de múltiples articuladores manuales y faciales tanto para la hipótesis de la vida diaria como para la hipótesis académica, en el último caso por lo menos en estos textos producidos por 
maestros sordos de básica primaria. De hecho, el presente estudio no permite afirmaciones contundentes, ya que se ha realizado con tres informantes sordos de la ciudad de Cali con características etarias y educativas particulares, incluyendo en cada caso un mayor o menor grado de oralización y, por consiguiente, un mayor o menor acceso a la lengua escrita académica. En realidad, como lo indican Lavid et al. (2010) o Lock (1996), la expresión de la hipótesis podría incluir múltiples recursos, difíciles de inventariar por completo. En particular, es posible que los rasgos no manuales innatos de origen subcortical y las reglas de supresión de gestos que cada hablante o señante aplica idiosincrásicamente no permitan nunca llegar a generalizaciones tajantes, sobre todo en el caso de las lenguas de señas. Este estudio se presenta, entonces, como una propuesta para que los mismos sordos produzcan y publiquen textos académicos que le sirvan de base a la comunidad para discutir la mejor manera de expresar contenidos académicos en la LSC en los diversos niveles, de modo que poco a poco se vaya configurando un estándar de LSC académica, incluyendo la expresión de la hipótesis.

A lo que sí tiene que acostumbrarse la comunidad sorda señante de LSC es que, con el aumento de los sordos en la educación media y superior, que trae consigo su acceso a lenguas escritas, sobre todo en nuestro medio al español, la lengua irá desarrollando un estándar académico que inevitablemente se verá influido por las formas del español u otras lenguas académicas escritas. En efecto, como en cualquier contacto interlingüístico e intercultural, más en áreas que no son de la experiencia cotidiana, algún tipo de influencia es inevitable, del mismo modo que en las lenguas modernas occidentales no se ha podido evitar la influencia, inicialmente del griego y del latín clásico y, más tarde, de otras lenguas modernas de mayor prestigio, en particular del inglés. El texto académico se lo puede clasificar como un "hecho social", en el sentido que le da al término Durkheim (1895/1982), por lo que ya trasciende fronteras políticas y lingüísticas. Y la LSC utilizada por miembros de la comunidad sorda que accedan a niveles cada vez más altos de escolaridad y de participación en la vida académica no puede ser la excepción.

\section{REFERENCIAS}

Austin, J.L. (1962). How to Do Things with Words. The William James Lectures delivered at Harward University 1955. http://dx.doi.org/10.1093/acprof:oso/9780198245537.001.0001.

Baker, C., y Padden, C. (1978). Focusing on the nonmanual components of American Sign Language. En P. Siple (Ed.), Understanding language through sign language research (pp. 27-57). Academic Press.

Baker, A., Van den Bogaerde, B., Pfau, R., y Schermer, T. (2016). The Linguistics of Sign Languages: An introduction. https://doi.org/10.1075/z.199.

Cooperreider, K., Abner, N., y Goldin-Meadow, S. (2018). The Palm-Up puzzle: Meanings and Origins of a Widespread Form in Gesture and Sign. Frontiers in Communication, 3. https://doi.org/10.3389/fcomm.2018.00023. 
Darwin, C. (1998). The expression of the emotions in man and animals ( $3^{\text {rd }}$ ed.). Oxford University Press.

De Bonnecaze, G., Vergez, S., Chaput, B., Vairel, B., Serrano, E., Chantalat, E., y Chaynes, P. (2019). Variability in facial-muscle innervation: A comparative study based on electrostimulation and anatomical dissection. Clinical Anatomy, 32(2), 169-175. https://doi.org/10.1002/ca.23081.

Durkheim, É. (1982). Las reglas del método sociológico. (Trad. L.E. Echevarría Rivera). Orbis. (Obra original publicada en 1895 bajo el título de Les règles de la méthode sociologique. Presses Universitaires de France).

Ekman, P. (2003). Emotions revealed: Recognizing Faces and Feelings to Improve Communication and Emotional Life. Times Books/Henry Holt and Company.

Ekman, P., y Friesen, W.V. (1975). Unmasking the face: A guide to recognizing emotions from facial clues. Prentice-Hall.

Ekman, P., y Friesen, W.V. (1976). Measuring facial movement. Environmental Psychology and Nonverbal Behavior, 1, 56-75. https://doi.org/10.1007/BF01115465.

Ekman, P., y Friesen, W.V. (1978). Facial Action Coding System: A technique for the measurement of facial movement. Consulting Psychologists Press.

Ekman, P., Friesen, W.V., y Hager, J.C. (2002). Facial Action Coding System: Manual and Investigator's Guide. Research Nexus.

Feyereisen, P. (1999). Neuropsychology of communicative movements. En L. Messing y R. Campbell (Eds.), Gesture, Speech and Sign (pp. 3-25). https://doi.org/10.1093/acprof:oso/9780198524519.003.0001.

Gothard K. M. (2014). The amygdalo-motor pathways and the control of facial expressions. Frontiers in neuroscience, 8. https://doi.org/10.3389/fnins.2014.00043.

Greimas, A.J. (1973). En torno al sentido: ensayos semióticos. (Trad. S. García Bardón). Fragua. (Obra original publicada en 1970 bajo el título de $\mathrm{Du}$ sens, essais sémiotiques. Éditions du Seuil).

Halliday, M.A.K. (2004). The language of science (The collected works of M. A. K. Halliday Vol. 5). (J.J. Webster, Ed.). Continuum.

Halliday, M.A.K. (2014). An introduction to functional grammar ( $4^{\text {th }}$ ed.) (Revised by C. Matthiessen). Routledge.

Hill, J.C., Lillo-Martin, D.C., y Wood, S.K. (2019). Sign languages: Structures and contexts. Routledge / Linguistic Society of America.

Hjelmslev, L. (1971). Prolegómenos a una teoría del lenguaje. (Trad. J.L. Díaz de Liaño Traducciones Diorki). Editorial Gredos. (Obra original publicada en 1943 bajo el título de Omkring sprogteoriens grundlæggelse. Bianco Lunos Bogtrykkeri).

Hjorstjö, C.-H. (1970). Man's face and mimic language. Studentlitteratur.

Johnson, R. (14-17 de noviembre de 2009). Conversations toward an international phonetic notation system for signed languages. Seminario en la Universidad Nacional, Bogotá D.C., Colombia.

Johnson, R. (27-29 de abril de 2010a). A course in sign language morphology. Seminario en la Universidad Nacional, Bogotá D.C., Colombia. 
Johnson, R. (24-26 de noviembre de 2010b). Practice in sign language phonetics. Taller. Bogotá D.C., Colombia.

Johnston, T., y Schembri, A. (2007). Australian Sign Language: An introduction to sign language linguistics. Cambridge University Press.

Kendon, A. (1975). Gesticulation, speech, and the gesture theory of language origins. Sign Language Studies, (9), 349-373.

Lavid, J.L., Arús, J., y Zamorano-Mansilla, J. (2010). Systemic Functional Grammar of Spanish: A Contrastive Study with English (Vols. I y II). Continuum.

Li Ching Ng, A., Rosenfeld, J.V., y Di Leva, A. (2019). Cranial Nerve Nomenclature: Historical Vignette. World Neurosurgery, 128, 299-307. https://doi.org/10.1016/j.wneu.2019.05.036.

Liddell, S.K. (1980). American Sign Language Syntax. Mouton Publishers.

Liddell, S.K. (2003). Grammar, Gesture and Meaning in American Sign Language. https://doi.org/10.1017/CBO9780511615054.

Liddell, S.K., y Johnson, R.E. (1989). American Sign Language: The Phonological Base. Sign Language Studies, (64), 195-278.

Lock, G. (1996). Functional English Grammar: An Introduction for Second Language Teachers. Cambridge University Press.

López, L., y M. Osorno. (2004). Tiempo, modo y aspecto en la Lengua de Señas Colombiana (LSC) [Tesis de Maestría inédita]. Universidad del Valle, Cali, Colombia.

Marieb, E. (2008). Anatomía y Fisiología Humana (9ª ed.). Pearson.

Matthiessen, C. (2004). Descriptive motifs and generalizations. En A. Caffarel-Cayron, J.R. Martin y C. Matthiessen (Eds.), Language Typology: A functional perspective (pp. 537-673). https://doi.org/10.1075/cilt.253.12mat.

McNeill, D. (1992). Hand and mind: What gestures reveal about thought. University of Chicago Press.

McNeill, D. (2005). Gesture and thought. https://doi.org/10.7208/chicago/9780226514642.001.0001.

Neidle, C., Kegl, J., MacLaughlin, D., Bahan, B., y Lee, R.G. (2000). The Syntax of American Sign Language: Functional Categories and Hierarchical Structures. MIT Press.

Oviedo, A. (2001). Apuntes para una gramática de la Lengua de Señas Colombiana. Universidad del Valle / Instituto Nacional para Sordos.

Oviedo, A. (2004). Classifiers in Venezuelan Sign Language. Signum.

Padden, C.A. (1988). Interaction of Morphology and Syntax in American Sign Language. Routledge.

Pfau, R., Steinbach, M., y Woll, B. (2012). Tense, aspect and modality. En R. Pfau, M. Steinbach y B. Woll (Eds.), Sign Language: An International Handbook (pp. 186204). https://doi.org/10.1515/9783110261325.186.

Raslan, A., Volk, G., Möller, M., Stark, V., Eckhardt, N., y Gutinas-Lichius, O. (2017). High variability of facial muscle innervations by facial nerve branches: A prospective electrostimulation study. The Laryngoscope, 127(6), 1288-1295. https://doi.org/10.1002/lary.26349. 
Rinn, W.E. (1991). Neuropsychology of facial expression. En R.S. Feldman y B. Rimé (Eds.), Fundamentals of Nonverbal Behavior (pp. 3-30). Cambridge University Press.

Sandler, W. (2012). Visual prosody. En R. Pfau, M. Steinbach y B. Woll (Eds.), Sign Language: An International Handbook (pp. 55-76). https://doi.org/10.1515/9783110261325.55.

Sandler, W., y Lillo-Martin, D. (2006). Sign Language and Linguistic Universals. Cambridge University Press.

Sato, W., Hyniewska, S., Minemoto, K., y Yoshikawa, S. (2019). Facial Expressions of Basic Emotions in Japanese Laypeople. Frontiers in Psychology, 10. https://doi.org/10.3389/fpsyg.2019.00259.

Sonne, J., Reddy, V., y Lopez-Ojeda, W. (9 de marzo de 2019). Neuroanatomy, Cranial Nerve $0 \quad$ (Terminal Nerve). StatPearls [Internet]. https://www.ncbi.nlm.nih.gov/books/NBK459159/.

Sutton-Spence, R., y Woll, B. (1998). The Linguistics of British Sign Language: An Introduction. Cambridge University Press.

Thompson, G. (2014). Introducing Functional Grammar (3 ${ }^{\text {rd }}$ ed.). Routledge.

Tomkins, S.S. (1962). Affect imagery consciousness. Vol. I. The positive affects. https://doi.org/10.1037/14351-000.

Tovar, L. (2006). Las unidades del análisis lingüístico en la modalidad viso-gestual. Lenguaje, 34, 15-70. https://doi.org/10.25100/lenguaje.v34i0.4832.

274 Tovar, L. (2008). Denominación, definición y creación de neologismos en la lengua de señas colombiana (LSC): Contribución a su planificación lingüística [Tesis de doctorado inédita]. Universidad de Los Andes, Mérida, Venezuela.

Tovar, L. (2017). La definición en la lengua de señas colombiana (LSC). Lenguaje, 45(2), 383-418. https://doi.org/10.25100/lenguaje.v45i2.5277.

Tovar, L., y López, L. (2018). La clasificación en la lengua de señas colombiana (LSC). Lenguaje, 46(1), 11-40. https://doi.org/10.25100/lenguaje.v46i1.6194.

Valli, C., Lucas, C., Mulrooney, K.J., y Rankin, M.N.P. (2011). Linguistics of American Sign Language: An Introduction ( $5^{\text {th }}$ ed.). Gallaudet University Press.

Wilbur, R.B. (1987). American Sign Language: Linguistic and applied dimensions (2 ${ }^{\text {nd }}$ ed.). Little, Brown and Company.

Wilbur, R.B. (1991). Intonation and focus in American Sign Language. En Y. No y M. Libucha (Eds.), ESCOL'90: Proceedings of the Seventh Eastern States Conference on Linguistics (pp. 320-331). Ohio State University Press.

Wilbur, R.B. (1994). Eyeblinks and ASL Phrase Structure. Sign Language Studies, (84), 221-240.

Wilcox, S., Rossini, P., y Antinoro, E. (2010). Grammaticalization in sign languages. En D. Brentari (Ed.), Sign languages (pp. 332-354). https://doi.org/10.1017/CBO9780511712203.016.

Zwirner, E., y Zwirner, K. (1937). Phonometrischer Beitrag zur Frage der Lesepausen. Archives Néerlandaises de Phonétique Expérimentale, (13), 111-128. 